\title{
Localized Plasmonic Resonances of Prolate Nanoparticles in a Symmetric Environment: Experimental Verification of the Accuracy of Numerical and Analytical Models
}

\author{
Mathias Kobylko, ${ }^{1}$ Pierre-Eugène Coulon, ${ }^{1}$ Abdallah Slablab, ${ }^{2}$ Alexandre Fafin, ${ }^{3}$ Julien Cardin, ${ }^{3}$ \\ Christian Dufour, ${ }^{3}$ Arthur Losquin, ${ }^{4}$ Mathieu Kociak, ${ }^{4}$ Isabelle Monnet, ${ }^{3}$ Dominique Mailly, ${ }^{5}$ Xavier Lafosse, ${ }^{5}$ \\ Christian Ulysse, ${ }^{5}$ Enric Garcia-Caurel, ${ }^{6}$ and Giancarlo Rizza ${ }^{1}$ \\ ${ }^{1}$ Laboratoire des Solides Irradiés (LSI), Ecole Polytechnique, CNRS (UMR 7642), \\ CEA, Université Paris-Saclay, 91128 Palaiseau Cedex, France \\ ${ }^{2}$ Laboratory of Photonics, Tampere University of Technology, FI-33101 Tampere, Finland \\ ${ }^{3}$ CIMAP, Normandie Université, ENSICAEN, UNICAEN, CEA, CNRS, \\ 6 Boulevard Maréchal Juin, 14050 Caen Cedex 4, France \\ ${ }^{4}$ Laboratoire de Physique des Solides (LPS), CNRS (UMR 8502), Université Paris-Sud, \\ Université Paris-Saclay, Bâtiment 510, Orsay, France \\ ${ }^{5}$ Centre de Nanosciences et de Nanotechnologies, CNRS, Université Paris-Sud, Université Paris-Saclay, \\ C2N-Marcoussis, 91460 Marcoussis, France \\ ${ }^{6}$ Laboratoire de Physique des Interfaces et des Couches Minces (LPICM), Ecole Polytechnique, CNRS, \\ Université Paris-Saclay, 91128 Palaiseau Cedex, France
}

\begin{abstract}
We study the evolution of the surface-plasmon resonances of individual ion-beam-shaped prolate gold nanoparticles embedded in a dielectric $\mathrm{SiO}_{2}$ environment by electron-energy-loss spectroscopy mapping in a scanning transmission electron microscope. The controlled symmetric dielectric environment obtained through the ion-beam-shaping method allows a direct quantitative comparison with numerical results obtained through simulations (auxiliary differential-equation finite-difference time-domain and boundary-element method) and with theoretical results obtained through analytical models (quasistatic model for prolate nanoellipsoids and waveguide model for infinite one-dimensional plasmonic waveguides), with which our experimental results are in very good agreement. We confirm the accuracy of state-of-the-art numerical tools and analytical theories that establish ion-beam shaping as a very promising method to design metal-dielectric nanocomposites with well-predicted optical properties, and with many possible applications in surfaceenhanced Raman spectroscopy and second-harmonic generation, as well as in conventional applications of metamaterials like negative refraction, superimaging, and invisibility cloaking.
\end{abstract}

\section{INTRODUCTION}

The ability to control and to confine light below the diffraction limit lies at the heart of the current interest in nanooptics. An approach consists in using the optical response of metallic nanoparticles (NPs) in metal-dielectric nanocomposites, which arises from resonances in the collective oscillations of the conduction electrons. These excitations, localized at the surface of metallic NPs, are called localized surface-plasmon resonances (LSPRs) [1-3]. One of their interesting features is their ability to focus light at the nanometer scale, leading to strong local fields (called hot spots) near the metal surfaces $[4,5]$. The fact that the energy of LSPRs can be tuned through variation of the shape, the size, the composition, or the environment of the metal NPs $[3,6,7]$ has opened the way to applications within the domains of biosensing [8], Raman spectroscopies [9,10], solar cells [11], near-field imaging [12], enhanced fluorescence spectroscopy [9], and nanolasers [13]. In addition, plasmonic excitations often play an important part in optical metamaterials [14-33] (i.e., artificially structured materials with a unit structure considerably smaller than the wavelength of visible light [34]), like those in negative optical index materials $[14,15]$, materials for superresolution applications [17-20], and electromagnetic cloaks [21].

In order to effectively design metal-dielectric nanocomposites for a particular application, the knowledge of the optical properties of the NPs at the nanometer scale is necessary. Near-field techniques, which include scanning optical microscopy $[35,36]$ and tip-enhanced photoluminescence [37], allow one to map local-field enhancements. However, these techniques have limited spatial and/or energy resolution. Conversely, electron-energy-loss spectroscopy in a scanning transmission electron microscope (STEM EELS) allows one to map optical properties at the nanoscale [38]. By raster scanning the electron beam over the metallic NPs, STEM EELS delivers information about the photonic local density of states of the metallic NPs in real space and energy space [39]. In other words, STEM 
EELS maps LSPRs at the nanometer scale in a broad spectral range [40].

LSPRs have been mapped by STEM EELS by several groups in NPs as different as nanotriangles [41], nanostars [42], ellipsoids [43,44], cubes [45], and nanowires [46-48]. Despite the large amount of literature, the quantitative agreement between experimental results and simulations or theories is often limited, for a major reason: the experiments are performed on NPs in a noncontrolled asymmetric dielectric environment. Indeed, NPs produced by conventional electron-beam lithography or chemical methods suffer from adhesion layers [49], surfactants [50], or simply the substrate by which they are supported [51,52]. All of these effects, which are more complicated to model, affect the optical properties of the NP and limit how simply and accurately one can predict and design metal-dielectric nanocomposites. In comparison, STEM-EELS experiments on simple NPs in a symmetric and controlled dielectric environment would allow testing the accuracy of state-of-the-art numerical tools and analytical theories. Moreover, such experiments, which require an elaborate fabrication method, would allow one to estimate how reliable the fabrication method is.

Ion-beam shaping is a technique with which one can shape nanostructures that are embedded in a dielectric matrix, while simultaneously imposing their orientation in space [53-77]. It has been well established that swift heavy-ion irradiation can be successfully used to elongate embedded nanoparticles along the direction of the incoming ion beam while maintaining a constant particle volume. Starting from easily fabricated near-spherical particles, one can then produce, in a controlled way, anisotropic nanorods or nanowires. Ion-beam irradiation thus allows the fabrication of encapsulated periodic arrangements of prolate NPs with controlled length-to-width ratios (aspect ratios), all oriented in a direction of choice, which are highly interesting for optical applications.

In this work, we use the ion-beam method to produce ideal model NPs in a symmetric environment and study them using STEM EELS. The STEM-EELS experiments allow us to track the evolution of the LSPRs with the shape of the NPs. We compare the experimental results to numerical auxiliary differential equation and finite-difference time-domain (ADE-FDTD) simulations, and boundary-element-method (BEM) simulations, as well as analytical solutions of Maxwell's equations within two different approximations (the quasistatic approximation for prolate nanoellipsoids and the waveguide approximation for infinite one-dimensional plasmonic waveguides). Our work confirms the accuracy of state-of-the-art numerical tools and analytical theories and establishes ion-beam shaping as a very promising method to design metaldielectric nanocomposites with well-predicted optical properties, and with many possible applications in surface-enhanced Raman spectroscopy and second-harmonic generation, as well as in conventional applications of metamaterials like negative refraction, superimaging, and invisibility cloaking.

\section{EXPERIMENTAL METHODS}

We use electron-beam lithography to fabricate Au NP arrays with a narrow size dispersion $(21.5 \pm 1.5 \mathrm{~nm}$ on most specimens) and a well-defined NP interdistance $(100 \mathrm{~nm})$ embedded within a dielectric silica $\left(\mathrm{SiO}_{2}\right)$ matrix. The precise steps of the Au-NP-array fabrication procedure, its parameters and further details can be found in Sec. I A of the Supplemental Material [78]. Additional details can be found in Ref. [79].

After the fabrication process, samples are irradiated with swift heavy ions to shape the NP morphology. The effects of swift heavy-ion irradiation on metallic NPs embedded in $\mathrm{SiO}_{2}$ have been studied by different groups [60,61,64,65,67] who observed that, during irradiation, the silica matrix dilates perpendicular to the beam direction, while the metallic NP deforms and elongates along the beam direction. Moreover, the deformation and elongation of the metallic NP depends on its initial size and shape, its chemical composition, the irradiation fluence, and the position where the NP is placed in the matrix with respect to the substrate [67].

Figures 1(a)-1(d) show the morphological evolution of $(21.5 \pm 1.5)-\mathrm{nm} \mathrm{Au}$ nanospheres as a function of the

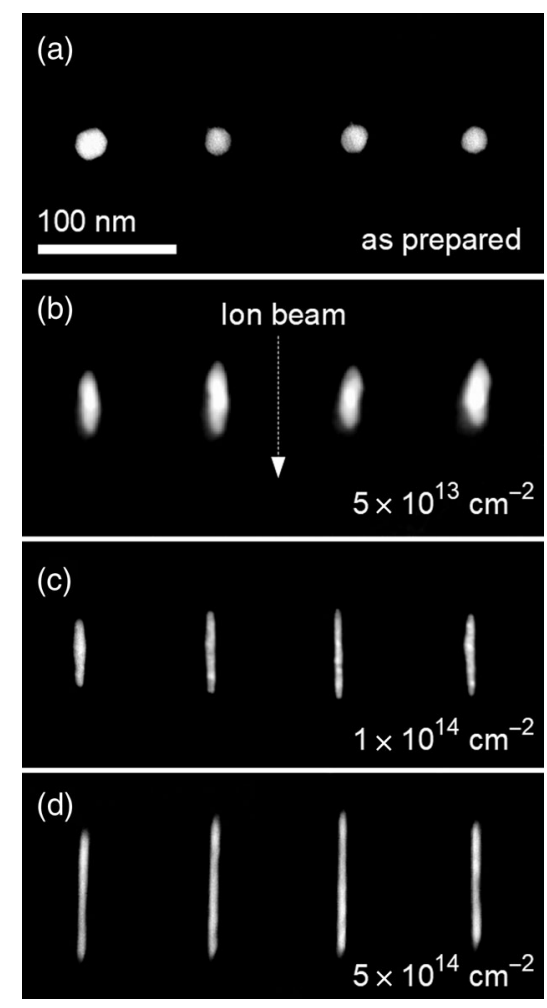

FIG. 1. STEM-HAADF images of gold NPs embedded into silica: (a) as initially prepared; after vertical swift heavy irradiation at fluences of (b) $5 \times 10^{13} \mathrm{~cm}^{-2}$, (c) $1 \times 10^{14} \mathrm{~cm}^{-2}$ and (d) $5 \times 10^{14} \mathrm{~cm}^{-2}$. 

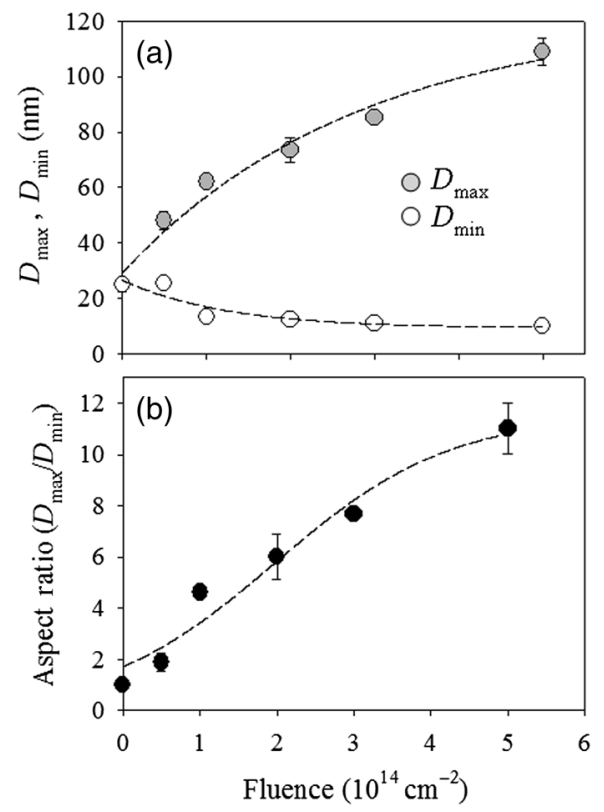

FIG. 2. Gold NPs embedded in silica after swift heavy irradiation at fluences between $5 \times 10^{13} \mathrm{~cm}^{-2}$ and $5 \times 10^{14} \mathrm{~cm}^{-2}$. (a) Dimensions. (b) Aspect ratio. The lines are guides for the eye.

irradiation fluence. Initially spherical NPs [see Fig. 1(a)] are continuously transformed into nanowires [see Fig. 1(d)] aligned along the beam direction. However, depending on the irradiation fluence, two intermediate shapes can be obtained: (i) faceted NPs at a fluence of $5 \times 10^{13} \mathrm{~cm}^{-2}$ to $1 \times 10^{14} \mathrm{~cm}^{-2}$ [see Figs. 1(b) and 2(b)] and (ii) prolate nanorods at a fluence of $1 \times 10^{14} \mathrm{~cm}^{-2}$ to $2 \times 10^{14} \mathrm{~cm}^{-2}$ [see Figs. 1(c) and 2(b)], to end up with nanowires at a fluence of $5 \times 10^{14} \mathrm{~cm}^{-2}$ [see Figs. 1(d) and 2(b)].

Figure 2(a) shows the evolution of both the NP length $\left(D_{\max }\right)$ and width $\left(D_{\min }\right)$ of prolate nanoparticles originating from $(21.5 \pm 1.5)-\mathrm{nm}$ Au nanospheres with the irradiation fluence. As the transformation takes place at constant volume, the elongation of the $\mathrm{NP}$ along the beam axis $\left(D_{\max }\right)$ is associated with a reduction of its width $\left(D_{\min }\right)$. For fluences larger than about 2 to $3 \times 10^{14} \mathrm{~cm}^{-2}$, both $D_{\max }$ and $D_{\min }$ reach a steady-state value, i.e., $110.1 \pm$ $5.2 \mathrm{~nm}$ for $D_{\max }$ and $9.9 \pm 1.0 \mathrm{~nm}$ for $D_{\min }$. However, for fluences higher than about 5 to $6 \times 10^{14} \mathrm{~cm}^{-2}$, the nanowires tend to become unstable and to break up into smaller fragments.

It has to be pointed out that the most appropriate parameter to study the evolution of the LSPR modes is not the irradiation fluence but the aspect ratio $\left(D_{\max } / D_{\min }\right)$. Thus, in Fig. 2(b), the relationship between these two parameters is reported. The aspect ratio increases slowly up to a fluence of approximately $1 \times 10^{14} \mathrm{~cm}^{-2}$. This corresponds to the transformation of the spheres into faceted NPs. However, for larger fluences, a rapid increase of the aspect ratio is observed. This increase corresponds to the transformation of the faceted NPs into nanorods and nanowires. Finally, for fluences larger than approximately 3 to $4 \times 10^{14} \mathrm{~cm}^{-2}$, the aspect ratio reaches a plateau at a value of approximately 11 .

It is worth noting that, because of their initial narrow size dispersion, all of the ion-shaped NPs in the array have nearly the same morphology for each irradiation fluence. Small fluctuations in the elongation rate are nonetheless observed [see Figs. 1(b)-1(d)], as prolate nanoparticles originating from smaller or bigger nanospheres, respectively, evolve further away or less far at the same fluence values (see previous work $[65,67,71]$ for a quantitative study of the shaping of gold nanoparticles by a swift heavyion beam).

We also observe that the pitch we choose for the array is sufficient to avoid any interaction among the NPs, i.e., their evolutions are independent of each other. Thus, for the study of the evolution of the LSPR modes, the evolution of a single NP is representative of the evolution of the ensemble of the NPs forming the array.

The parameters and technical details of the NP shaping by ion-irradiation procedure can be found in Sec. I B of the Supplemental Material [78]. Additional details can be found in Refs. [60,61,64,65,67,80].

The irradiated Au NP arrays are then prepared in a crosssection geometry by a standard focused-ion-beam (FIB) technique and mounted on a STEM operating at $100 \mathrm{kV}$ and fitted with a custom scanning and spectral imaging unit, allowing simultaneous high-angle annular dark-field (HAADF) imaging and EELS spectrum acquisition. From a classical electrodynamics perspective, electrons from the incident STEM electron beam excite NP plasmons due to the coupling of their electromagnetic field to the plasmonic evanescent-field components close to the NP surface. As the excitation of a plasmon leads to an energy loss equal to the energy transferred to the plasmon, the EELS spectrum contains resonances that reflect the plasmons which are excited at the position of the beam. Spatially resolved experiments are performed by scanning the electron beam over the sample and by recording a full EELS spectrum for each electron-beam position. EELS maps are then obtained by plotting the spatial variations of the EELS signal filtered for a given resonance energy. As a consequence, the EELS maps show the spatial variations of the plasmon mode that is resonant at this energy [40]. Further details of the plasmon mapping procedure can be found in Sec. IC of the Supplemental Material [78]. Additional details can be found in Ref. [81].

\section{NUMERICAL METHODS AND THEORY}

We compare these experimental results to numerical ADE-FDTD simulations and to BEM simulations, as well as to analytical solutions of Maxwell's equations within two different approximations (the quasistatic approximation for prolate nanoellipsoids and the waveguide 
approximation for infinite one-dimensional plasmonic waveguides). The NP dimensions necessary for these simulations are determined from the HAADF images.

The ADE-FDTD method is a powerful method to simulate electromagnetic wave propagation in dispersive materials with arbitrary geometries and has proven to be well adapted to different kinds of numerical spectroscopic studies, particularly in plasmonics [82-84]. Further details can be found in Sec. II A of the Supplemental Material [78]. Additional details can be found in Refs. [85-88]. In the following, we use the ADE-FDTD method to simulate the electric-field enhancement induced by a plane-wave excitation on the NP.

In all of the electric-field enhancement maps simulated by ADE FDTD, the volume of the nanoparticles is discretized within the staircase approximation with a space step of $0.4 \mathrm{~nm}$ (except in the case of the spherical NP, where the space step is $0.2 \mathrm{~nm}$ ). The energy imprecision of the ADE-FDTD calculations is approximately $0.02 \mathrm{eV}$. (However, this imprecision does not take into account any possible imprecisions of the used dielectric function itself.) In almost all of the simulations, the electric field $\mathbf{E}$ of the incident plane wave is parallel to the long axis of the nanoparticle. The only exceptions are the maps showing either transverse modes or longitudinal plasmonic modes with a zero dipole moment which cannot be excited in this geometry. Thus, in these maps, the electric field $\mathbf{E}$ of the incident plane wave creates a $90^{\circ}$ angle $\left(45^{\circ}\right.$ angle) for the excitation of the transverse modes (the excitation of the longitudinal plasmonic modes with a zero dipole moment) with the long axis of the nanoparticle.

The BEM method allows us to calculate the plasmonic excitation of the Au NP in response to the incident electron beam of the STEM. The MNPBEM code developed by Hohenester and Trügler [89-91], which in recent years has been extensively used for the simulation of plasmonic nanoparticles [92-108], is used for these simulations. Further details can be found in Sec. II B of the Supplemental Material [78]. Additional details can be found in Refs. [89-91,109,110]. We use the BEM method to simulate the EELS experiments.

In all of the EELS maps simulated by MNPBEM, the surfaces of the four NPs are discretized through triangulation. The size of the triangles (for the sphere and the faceted nanoparticle) and the quadrilaterals (for the nanorod and the nanowire) varies between approximately $1 \mathrm{~nm}$ (on curved surface parts) and approximately $3 \mathrm{~nm}$ (on flat surface parts). The positions of the vertices of the triangles and quadrilaterals in space are not discretized. This way, the dimensions and the overall surface of all four NPs are reproduced within a precision of $0.1 \mathrm{~nm}$. (An exception is the triangulated surface of the faceted NP, which presents a 1-nm large irregularity at one of its ends.) The energy imprecision of the MNPBEM calculations is approximately $0.01 \mathrm{eV}$. (However, this imprecision does not take into account any possible imprecisions of the used dielectric function itself.)
In order to ascertain a better comparability, the same dielectric functions are used for the ADE-FDTD simulations, the MNPBEM simulations, and the two analytical models (the quasistatic model and the waveguide model). In the ADE-FDTD method, the permittivity of a dispersive material is described by a superposition of several oscillators described by the Debye, the Drude, or the Lorentz dispersion model. In order to describe the gold refractive index tabulated by Palik [111] between 0.5 and $3 \mathrm{eV}$, we fit these data by one Drude oscillator and two Lorentz oscillators. The numerical values of the parameters as well as the graphs of the resulting dielectric functions are shown in Sec. II C in the Supplemental Material [78]. Additional details can be found in Refs. [2,111,112].

When comparing electric-field enhancement maps simulated by ADE-FDTD and EELS maps simulated by MNPBEM to each other as well as to experimental STEM-EELS maps, one has to be aware of three main differences between these two simulation methods.

First, in contrast to ADE-FDTD simulations, the MNPBEM simulations show not the electric-field enhancement but the probability to excite a plasmon of a particular energy by placing a focused high-energy electron beam at a particular location on or next to the specimen, as is also the case in the experimental STEM-EELS map. Second, in contrast to ADE-FDTD simulations, which use a plane electromagnetic wave as excitation, in the MNPBEM simulations, for each image point, a localized electron beam at the same point (generating an electromagnetic wave which is more similar to dipole radiation than to a plane electromagnetic wave [113]) is used as excitation of the plasmonic resonance (i.e., the source of excitation is different for each image point). Third, resonance is defined differently in each of the two simulation methods: in the ADE-FDTD simulations, the energy at which the total normalized electric field $|\mathbf{E}| /\left|\mathbf{E}_{0}\right|$ is maximized in an arbitrary point of the specimen is taken as the resonance energy, whereas, in the MNPBEM simulations, the energy at which the plasmonic excitation probability is maximized at an arbitrary point of the specimen is taken as the resonance energy.

Therefore, the electric-field enhancement maps simulated by ADE FDTD and the EELS maps simulated by MNPBEM are complementary. The EELS maps simulated by MNPBEM allow for a more direct comparison with the experimental EELS maps, whereas the electric-field enhancement maps simulated by ADE FDTD allow one to make better predictions of the optical properties of the studied NPs. As both maps show different quantities, we expect them to differ, and we do not expect precise quantitative agreement between the plasmonic resonance energies obtained by these two simulation methods for all possible NP geometries, either, even if for simple NP geometries (as studied here) the quantitative agreement seems to be very good (see Table I). 
TABLE I. Experimental and numerical values of the energies of the plasmonic resonances.

\begin{tabular}{|c|c|c|c|c|c|c|c|c|}
\hline \multicolumn{3}{|c|}{ Plasmonic resonance } & \multicolumn{3}{|c|}{ Energy $(\mathrm{eV})$} & \multicolumn{3}{|c|}{ Energy differences $(\mathrm{eV})$} \\
\hline Particle & Mode & Harmonic & EELS & $\begin{array}{c}\text { ADE } \\
\text { FDTD }\end{array}$ & MNPBEM & $\begin{array}{c}\text { ADE } \\
\text { FDTD EELS }\end{array}$ & $\begin{array}{l}\text { MNPBEM } \\
\text { EELS }\end{array}$ & $\begin{array}{c}\text { MNPBEM } \\
\text { ADE FDTD }\end{array}$ \\
\hline Nanosphere (round) & Dipolar & & & 2.30 & 2.39 & & & 0.09 \\
\hline Nanosphere $(\mathcal{A}=1.10)$ & Dipolar & & 2.22 & 2.27 & 2.35 & 0.05 & 0.13 & 0.08 \\
\hline Faceted NP & Longitudinal & & 1.90 & 1.78 & 1.94 & -0.12 & 0.04 & 0.16 \\
\hline Faceted NP & Transverse & & 2.33 & 2.29 & 2.31 & -0.04 & -0.02 & 0.02 \\
\hline Nanorod & Longitudinal & First & 1.18 & 1.24 & 1.29 & 0.06 & 0.11 & 0.05 \\
\hline Nanorod & Longitudinal & Second & 1.80 & 1.91 & 1.95 & 0.11 & 0.15 & 0.04 \\
\hline Nanorod & Interband & & 2.45 & 2.54 & 2.60 & 0.09 & 0.15 & 0.06 \\
\hline Nanowire & Longitudinal & First & 0.86 & 0.67 & 0.67 & -0.19 & -0.19 & 0.00 \\
\hline Nanowire & Longitudinal & Second & 1.27 & 1.15 & 1.17 & -0.12 & -0.10 & 0.02 \\
\hline Nanowire & Longitudinal & Third & 1.66 & 1.46 & 1.53 & -0.20 & -0.13 & 0.07 \\
\hline Nanowire & Interband & & 2.40 & 2.60 & 2.60 & 0.20 & 0.20 & 0.00 \\
\hline
\end{tabular}

\section{EXPERIMENTAL AND SIMULATED PLASMONIC PROPERTIES OF THE SHAPED PARTICLES}

In this section, we investigate the evolution of the LSPR modes as a function of the shape and the rising aspect ratio (nanosphere, faceted nanoparticle, nanorod, and nanowire), which are directly related to the irradiation fluence [see Fig. 2(b)]. For each nanoparticle type, experimental EELS maps obtained from the STEM-EELS analysis are compared to EELS maps simulated by MNPBEM and to electricfield enhancement maps simulated by ADE FDTD.

The evolution of the shape of the gold NPs takes place at a constant volume. However, the study of the plasmonic response is performed on NPs which evolve from different initial volumes. The reason is that the preparation of thin cross sections (with an approximate thickness of $0.1 \mu \mathrm{m}$ ) out of the irradiated specimen, which is necessary for the plasmon mapping in a STEM (see Sec. IC in the Supplemental Material [78]), makes the specimen unsuitable for further irradiation, where a thick specimen is required to ascertain the isotropy of the system in the plane perpendicular to the irradiation direction (see Sec. I B in the Supplemental Material [78]).

The measurement uncertainties of the NPs dimensions given in the following sections are based on the measurement imprecision of these values on the STEM-HAADF images of the NPs. In addition, all of these values are subject to an additional bias of $\pm 10 \%$ caused by the calibration imprecision of the electron microscope. This additional bias affects the absolute size of the NPs but not their aspect ratios, and it is not mentioned explicitly in the following sections. The energy resolution of the EELS spectra differs between different nanoparticles and lies between approximately 0.10 and $0.15 \mathrm{eV}$.

\section{A. Experimental EELS maps (STEM EELS)}

The typical shape of nonirradiated nanoparticles is spherical with an aspect ratio of $1.0 \pm 0.1$ [see Fig. 2(b)]. As an example, Fig. 3(a) shows the HAADF-STEM image of an isolated $\mathrm{Au} \mathrm{NP}$ of dimensions $(24.4 \pm 0.6 \mathrm{~nm}) \times$ $(22.1 \pm 0.6 \mathrm{~nm})$. This Au NP is approximately spherical (aspect ratio, $1.10 \pm 0.05$ ) and its volume is equal to that of a sphere with a diameter of $22.8 \pm 0.6 \mathrm{~nm}$.

The corresponding integrated EELS spectrum is shown in Fig. 3(b). It is obtained by summing up all of the individual EELS spectra acquired over the NP (see Sec. IC in the Supplemental Material [78]). It unveils an intense peak at the energy of $2.22 \mathrm{eV}$. This resonance corresponds to the LSPR dipolar mode of a gold NP embedded within a silica matrix. In other words, the observed resonance is associated with the fulfillment of the Fröhlich condition, $\varepsilon_{\mathrm{Au} 1}=-2 \varepsilon_{\mathrm{SiO}_{2} 1}$, where $\varepsilon_{\mathrm{Au} 1}$ is the real part of the dielectric function of the $\mathrm{Au} \mathrm{NP}$ and $\varepsilon_{\mathrm{SiO}_{2} 1}$ the real part of the dielectric function of the embedding silica medium. The experimental EELS map corresponding to the dipolar resonance in the integrated EELS spectra is shown in Fig. 3(c). Because of the almost-spherical symmetry of the NP, the dipolar LSPR is almost homogeneously distributed over the surface of the sphere (in STEM EELS, the source of excitation is different for each image point). The asymmetries in the EELS map are attributed to imperfections of the spherical NP and to the slight deviation from the spherical shape.

An irradiation of spherical NPs by the swift heavy-ion beam results first in their transformation into prolate faceted NPs with an aspect ratio of roughly $2 \pm 0.5$ [see Figs. 1(b) and 2(b)]. The prolate faceted NP shown in Fig. 4(a) has a length of $61.6 \pm 2.1 \mathrm{~nm}$ and a width of $31.4 \pm 1.0 \mathrm{~nm}$, and thus an aspect ratio of $1.96 \pm 0.14$. 

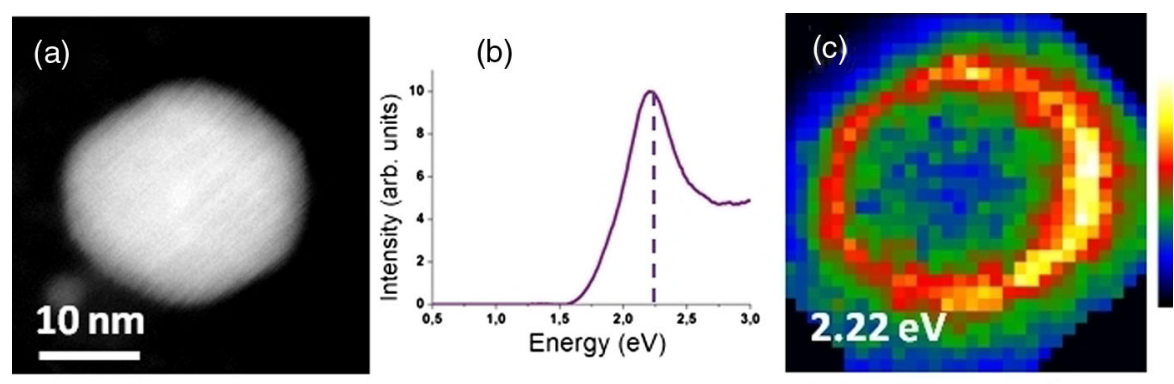

FIG. 3. Nanosphere. (a) STEMHAADF image. (b) EELS spectrum. (c) Experimental EELS map (obtained by STEM EELS). (d) Simulated electricfield enhancement map (obtained by ADE FDTD). (e) Simulated EELS map (obtained by MNPBEM).

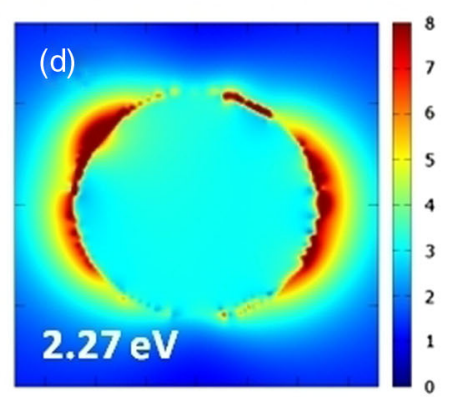

For nonspherical NPs in the dipole limit $(D \ll \lambda)$, the resonance condition is modified to account for the change in boundary conditions at the NP surface. For spheroidlike NPs, this change results in long-axis (longitudinal) and short-axis (transverse) dipole plasmon modes which are shifted with respect to the plasmon dipole resonance frequency (energy) of a spherical NP. The longitudinal mode is shifted towards lower energies and the transverse mode towards higher energies.

In the integrated EELS spectrum, the longitudinal and transverse plasmon modes are observed, respectively, at 1.90 and $2.33 \mathrm{eV}$ [see Fig. 4(b)]. The corresponding
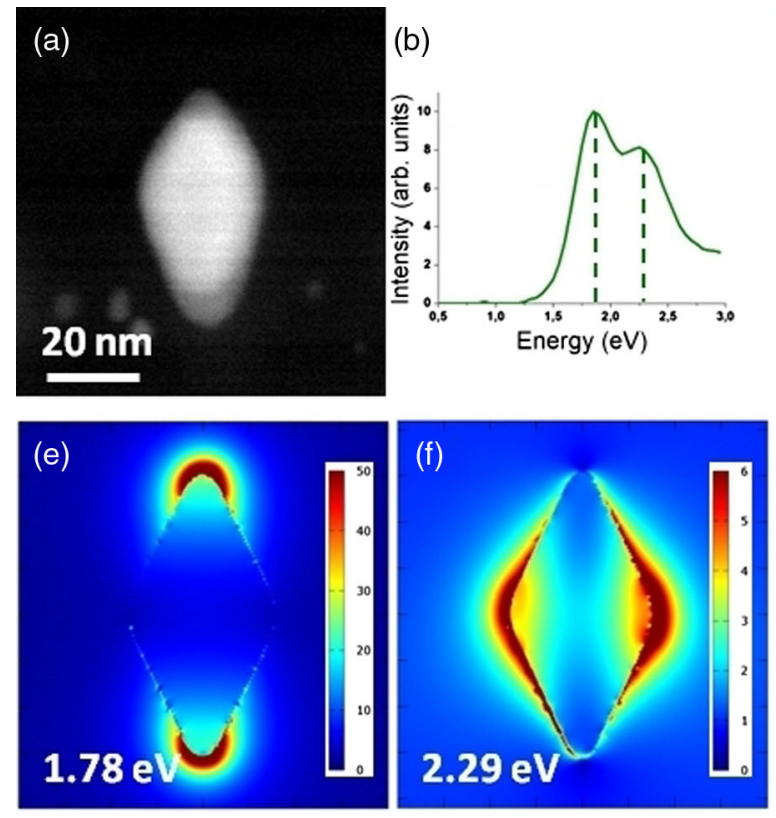

experimental EELS maps are shown in Figs. 4(c) and 4(d) for the two resonances. The longitudinal mode is parallel to the major axis (i.e., localized at the poles of the NP), whereas the transverse mode is parallel to the minor axis (i.e., localized at the equatorial region).

When faceted NPs are subjected to more irradiation by the swift heavy-ion beam, they transform into nanorods aligned along the beam direction with approximate aspect ratios of $5 \pm 1$ [see Figs. 1(c) and 2(b)]. The STEMHAADF image in Fig. 5(a) shows a $\mathrm{Au}$ nanorod with a length of $51.1 \pm 1.2 \mathrm{~nm}$ and a width of $11.2 \pm 1.5 \mathrm{~nm}$, and thus an aspect ratio of $4.6 \pm 0.8$.
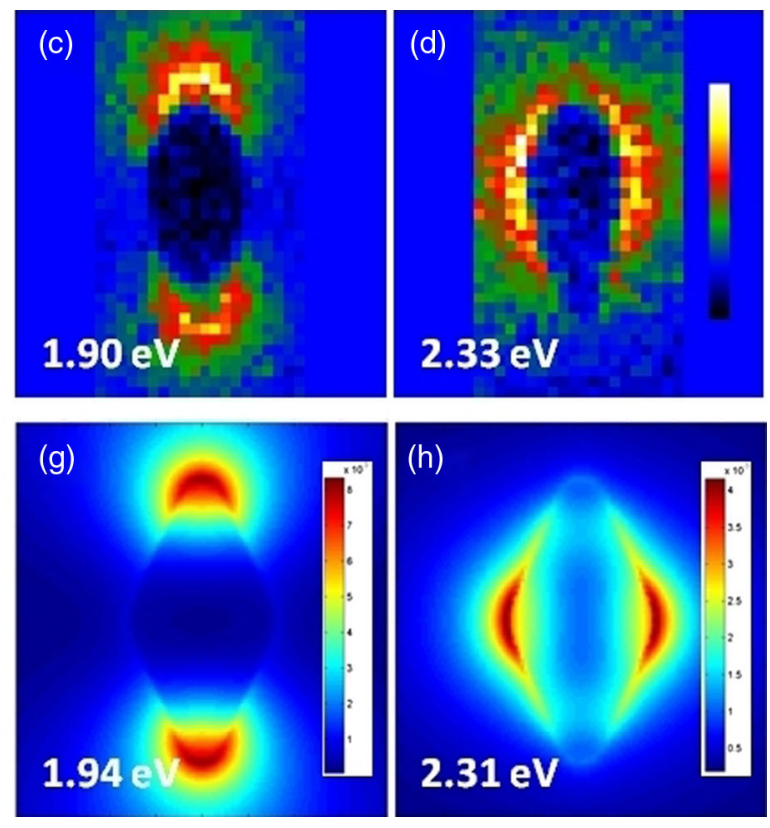

FIG. 4. Prolate faceted nanoparticle. (a) STEM-HAADF image. (b) EELS spectrum. (c),(d) Experimental EELS maps (obtained by STEM EELS). (e),(f) Simulated electric-field enhancement map (obtained by ADE FDTD). (g),(h) Simulated EELS map (obtained by MNPBEM). 


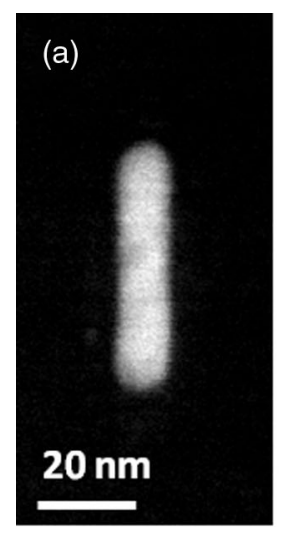

(b)
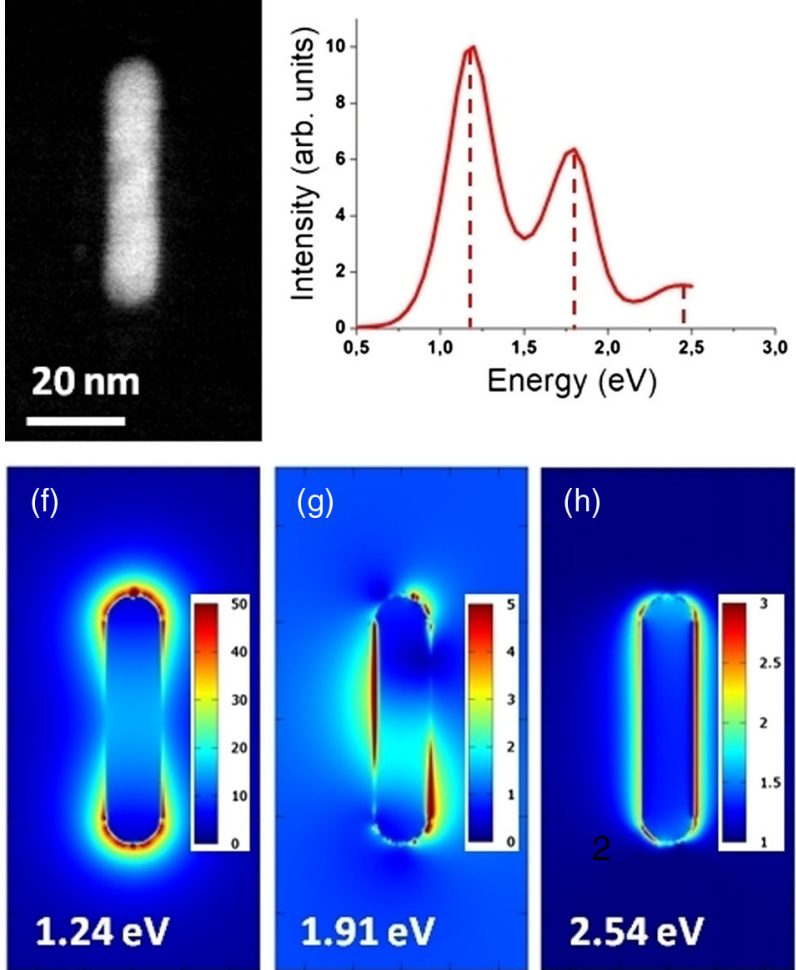

\section{(g)}

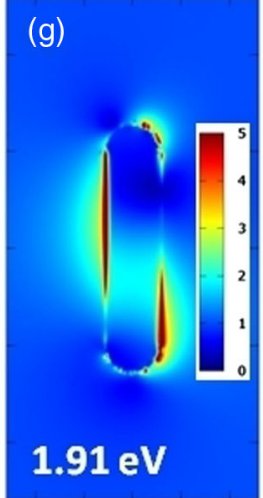

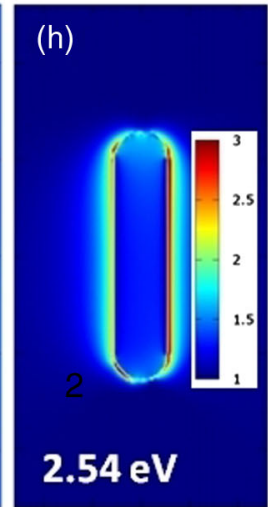
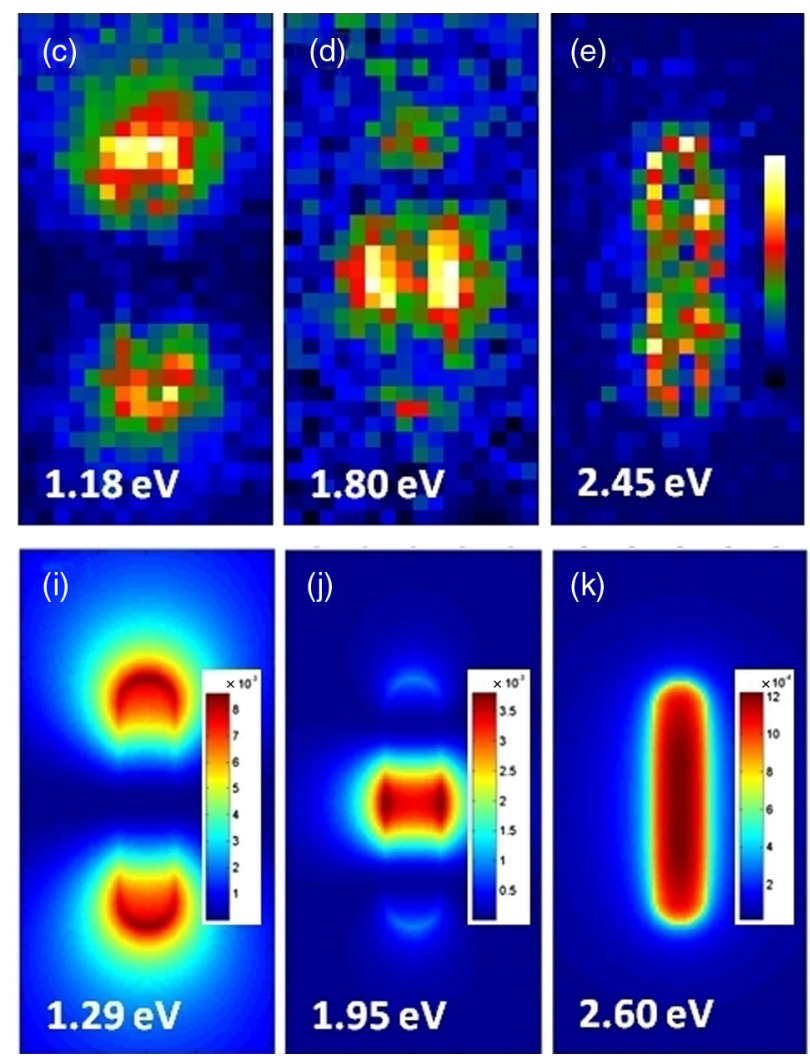

FIG. 5. Nanorod. (a) STEM-HAADF image. (b) EELS spectrum. (c)-(e) Experimental EELS map (obtained by STEM EELS). (f)-(h) Simulated electric-field enhancement map (obtained by ADE FDTD). (i)-(k) Simulated EELS map (obtained by MNPBEM).

The corresponding averaged EELS spectrum [see Fig. 5(b)] reveals two strong resonance peaks, at 1.18 and $1.80 \mathrm{eV}$, and a weak resonance peak at $2.45 \mathrm{eV}$. The experimental EELS maps associated with the distinct plasmon-mode peak resonance energies are shown in Figs. 5(c)-5(e). The EELS map at $1.18 \mathrm{eV}$ in Fig. 5(c) corresponds to the first longitudinal mode (dipolar mode), whereas the EELS map at $1.80 \mathrm{eV}$ in Fig. 5(d) is the second longitudinal harmonic. Analogous to the faceted prolate particle in Fig. 4, one would expect the weak resonance at $2.45 \mathrm{eV}$ in Fig. 5(e) to be identified as the transverse mode of the particle. However, owing to the onset of interband transitions at the same energy (see Sec. IIC in the Supplemental Material [78]), the observed weak resonance peak at $2.45 \mathrm{eV}$ cannot be ascribed to the transverse mode.

A further increase of the fluence transforms the nanorods into nanowires of approximate aspect ratios of $9 \pm 3$ [see Figs. 1(d) and 2(b)], or even higher for nanorods which are initiated from nanospheres significantly bigger than $21.5 \pm 1.5 \mathrm{~nm}$. The STEM-HAADF image in Fig. 6(a) shows a Au nanowire with a length of $92.6 \pm 3.3 \mathrm{~nm}$ and a width of $7.8 \pm 1.3 \mathrm{~nm}$, and thus an aspect ratio of $11.9 \pm 2.9$. [Based on its volume, we conclude that it develops from a nanosphere of diameter $20.4 \pm 2.6 \mathrm{~nm}$, which is considerably lower than the nanosphere the prolate faceted nanoparticle develops from (a diameter of
$33.8 \pm 1.1 \mathrm{~nm}$ ). Thus, it (as expected) already reaches the nanowire shape at a fluence of $5 \times 10^{14} \mathrm{~cm}^{-2}$.]

The corresponding averaged EELS spectrum [see Fig. 6(b)] reveals four resonance peaks at the following eigenmode energies: $0.86,1.27,1.66$, and $2.40 \mathrm{eV}$. The experimental EELS maps associated with the distinct plasmon-mode peak resonance energies are displayed in Figs. 6(c)-6(f). The first EELS map at $0.86 \mathrm{eV}$ in Fig. 6(c) corresponds to the first longitudinal mode (the dipolar mode). The second and third maps at 1.27 and $1.66 \mathrm{eV}$ in Figs. 6(d) and 6(e) are, respectively, the second and third longitudinal harmonics.

Analogous to the faceted prolate particle in Fig. 4, one would expect the weak resonance at $2.40 \mathrm{eV}$ in Fig. 6(f) to be identified as the transverse mode of the particle. However, owing to the onset of interband transitions at the same energy (see Sec. II C in the Supplemental Material [78]), the observed weak resonance peak at $2.40 \mathrm{eV}$ cannot be ascribed to the transverse mode. As this is already the case for the nanorod (see Fig. 5), it is also to be expected for the nanowire as, due its higher aspect ratio $(11.9 \pm 2.9$, as opposed to $4.6 \pm 0.8$ for the nanorod), its transverse mode is expected to be even more blueshifted with respect to the dipolar mode of the nanosphere than the transverse mode of the nanorod. 

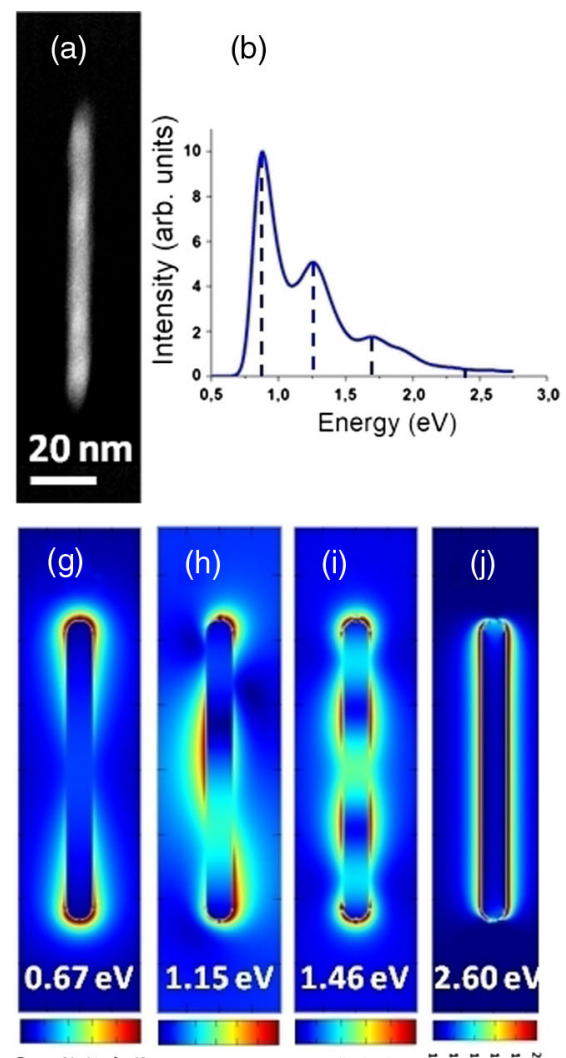

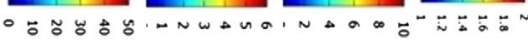
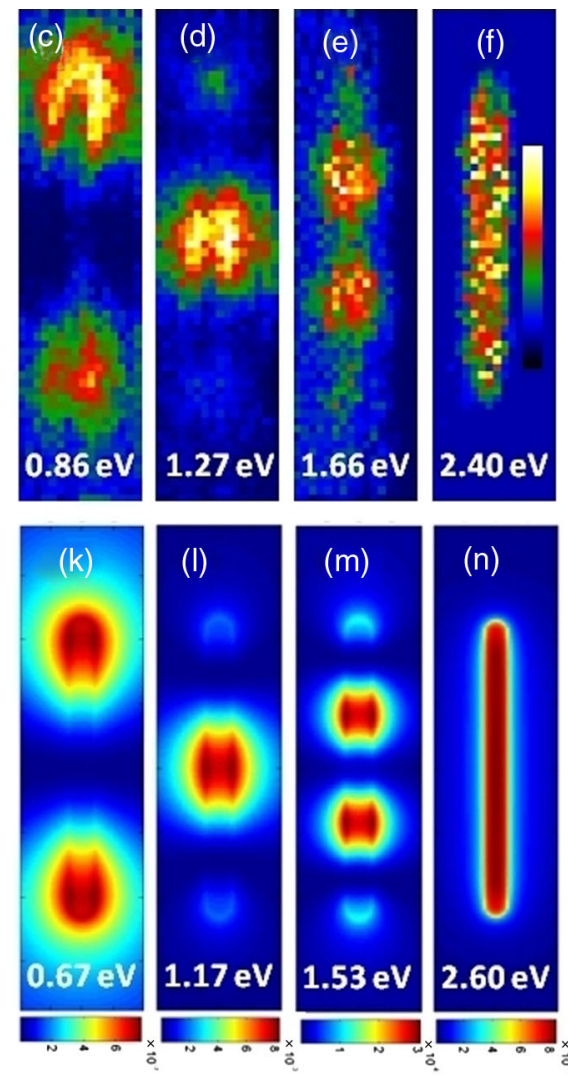

FIG. 6. Nanowire. (a) STEM-HAADF image. (b) EELS spectrum. (c)-(f) Experimental EELS map (obtained by STEM EELS). (g)-(j) Simulated electric-field enhancement map (obtained by ADE FDTD). (k)-(n) Simulated EELS map (obtained by MNPBEM).

\section{B. Simulated EELS maps (MNPBEM)}

In order to simulate the EELS map of the spherical Au NP in Fig. 3, this NP is modeled as a perfect sphere with a diameter of $22.8 \mathrm{~nm}$ (not shown) and as an ellipsoid with the three axes $24.4 \times 22.1 \times 22.1 \mathrm{~nm}$ (aspect ratio, 1.10) in the MNPBEM simulations.

The MNPBEM simulations in Fig. 3(e) show a simulated EELS map of a plasmonic excitation at a resonance energy of $2.22 \mathrm{eV}$. The plasmonic excitation is almost homogeneously distributed over the surface of the Au nanoellipsoid with a 1.10 aspect ratio and can be identified as the dipolar mode of this NP. [In experimental as well as in simulated EELS maps, the source of excitation is different for each image point (see Sec. III for further details), which leads to a homogeneous distribution of the dipolar mode in the case of a perfectly spherical (or close to perfectly spherical) particle.]

The faceted prolate nanoparticle in Fig. 4 is modeled as an octahedron covered with an adaptive surface mesh using the DistMesh mesh-generation script [114] enhanced with custom scaled edge-length functions so that the mesh density is higher at the corners and edges and lower at the flat surfaces, and with custom signed distance functions so that all of the edges and corners are rounded. An alternative way to obtain the same octahedron is to start with a regular octahedron in which all three axes equal $100 \mathrm{~nm}$ and to roll a sphere with a 21-nm radius over the entire surface. The surface on which the center of this sphere moves defines a new $(142 \times 142 \times 142)$-nm octahedron with rounded corners and edges. By contracting this octahedron by a factor of $61.6 \mathrm{~nm} / 142 \mathrm{~nm}$ in one dimension and by the factor of $31.4 \mathrm{~nm} / 142 \mathrm{~nm}$ in the other two dimensions, one obtains the octahedron used for the MNPBEM simulations, which has the dimensions $62.0 \times$ $31.5 \times 31.5 \mathrm{~nm}$ after a rounding of the edges and corners.

The MNPBEM simulations in Figs. 4(g) and 4(h) show simulated EELS maps at plasmonic resonance energies of 1.94 and of $2.31 \mathrm{eV}$, respectively, which can be identified as the longitudinal and the transverse mode of the prolate faceted $\mathrm{Au}$ nanoparticle.

The nanorod in Fig. 5 is modeled as a cylinder terminated on both ends by a hemispherical cap. The diameter of the cylinder is $11.2 \mathrm{~nm}$ and the length of the total structure (including the hemispherical caps) is $51.1 \mathrm{~nm}$. The MNPBEM simulations in Figs. 5(i) and 5(j) show simulated EELS maps at the plasmonic resonance energies of 1.29 and $1.95 \mathrm{eV}$, respectively, which can be identified as the first longitudinal mode (the dipolar mode) and as the second longitudinal harmonic of the Au nanorod, respectively. Analogous to the faceted prolate particle in Fig. 4, one would expect the resonance at $2.60 \mathrm{eV}$ in Fig. 5(e) to be identified as the transverse mode of the particle. However, owing to the onset of interband transitions at the same energy (see Sec. IIC in the Supplemental Material [78]), the observed resonance peak at $2.60 \mathrm{eV}$ cannot be ascribed to the transverse mode. 
The nanowire in Fig. 6 is modeled as a cylinder terminated on both ends by a hemispherical cap. The diameter of the cylinder is $7.8 \mathrm{~nm}$ and the length of the total structure (including the hemispherical caps) is $92.6 \mathrm{~nm}$. The MNPBEM simulations in Figs. 6(k)-6(m) show simulated EELS maps at plasmonic resonance energies of $0.67,1.17$, and $1.53 \mathrm{eV}$, respectively, which can be identified as the first longitudinal mode (the dipolar mode), the second longitudinal harmonic, and the third longitudinal harmonic of the Au nanowire. As for the nanorod, owing to the onset of interband transitions (see Sec. II C in the Supplemental Material [78]), the observed resonance peak at $2.60 \mathrm{eV}$ in Fig. 6(n) cannot be ascribed to the transverse mode.

\section{Simulated electric-field enhancement maps (ADE FDTD)}

The optical behavior of the spherical Au NP in Fig. 3 is modeled using a simple spherical geometry for a spherical NP with a diameter of $22.8 \mathrm{~nm}$ (not shown), and a simple ellipsoidal geometry for an ellipsoidal NP with the three axes $24.4 \times 22.1 \times 22.1 \mathrm{~nm}$ (and thus with an aspect ratio equal to 1.10). The modification of the local field induced by this NP when embedded in a silica matrix is simulated with the ADE-FDTD code, and the results for the ellipsoidal NP are shown in Fig. 3(d). Here, the field distribution is represented as the ratio between the amplitude of the electric field with and without the NP, i.e., $|\mathbf{E}| /\left|\mathbf{E}_{0}\right|$. It is worth noting that, due to electric-field polarization, the field intensity $\left(|\mathbf{E}| /\left|\mathbf{E}_{0}\right|\right)$ is more important at the poles. The maximum of the intensity, i.e., the resonant dipolar mode, is obtained for an energy of $2.27 \mathrm{eV}$. The irregularities in the ADE-FDTD map are most probably due to the staircase approximation.

The real morphology of the ion-shaped faceted NP in Fig. 4 is quite complicated. Thus, for the ADE-FDTD simulations, it is approximated by an octahedron with rounded corners and edges (see Sec. IV B for further details). ADE-FDTD simulations are performed for a NP having a major axis of $61.6 \mathrm{~nm}$ and two minor axes of $31.4 \mathrm{~nm}$. We obtain the values of $1.78 \mathrm{eV}$ for the longitudinal mode and $2.29 \mathrm{eV}$ for the transverse mode [see Figs. 4(e) and 4(f)].

We perform ADE-FDTD simulations for the Au nanorod in Fig. 5 with a length of $51.1 \mathrm{~nm}$ and a width of $11.2 \mathrm{~nm}$ using a straight cylinder terminated by hemispherical caps. Figures 5(f)-5(h) show the field distributions at the peak maxima found at the energy positions of 1.24, 1.91, and approximately $2.54 \mathrm{eV}$. It has to be pointed out that the second longitudinal harmonic shown in Fig. 5(g) has a zero dipole moment, and that it is thus excited with the electric field $\mathbf{E}$ of the incident plane wave creating a $45^{\circ}$ angle with the long axis of the nanoparticle, which results in the noticeable asymmetry between the left and the right part of Fig. 5(g).
A straight-cylinder geometry terminated by hemispherical caps is also used for the ADE-FDTD simulations for the $\mathrm{Au}$ nanowire in Fig. 6, only this time with a length of $92.6 \mathrm{~nm}$ and a width of $7.8 \mathrm{~nm}$. Figures $6(\mathrm{~g})-6(\mathrm{j})$ show the field distributions at the peak maxima found at the energy positions of $0.67,1.15,1.46$, and approximately $2.60 \mathrm{eV}$.

As is the case in Fig. 5(g) for the nanorod, the second longitudinal harmonic of the nanowire shown in Fig. 6(h) also has a zero dipole moment. It is thus excited with the electric field $\mathbf{E}$ of the incident plane wave creating a $45^{\circ}$ angle with the long axis of the nanoparticle, which results in the noticeable asymmetry between the left and the right part of Fig. 6(h).

Overall, our results show the shifts in resonance energy due to NP length (e.g., Fig. 3 versus Figs. 4-6). As expected [47], the mode energy decreases with an increasing aspect ratio, and higher harmonic modes emerge as the rod aspect ratio increases.

\section{Comparison of simulations and experiments}

Table I summarizes the experimental and numerical values of the energies of the plasmonic resonances.

\section{Nanosphere}

Both the ADE-FDTD simulations for a perfect sphere $(2.30 \mathrm{eV})$ and the MNPBEM simulations for a perfect sphere $(2.39 \mathrm{eV})$ return energy values for the plasmon resonance energy which are blueshifted with respect to the experimental one $(2.22 \mathrm{eV})$. By taking into account that the nanosphere deviates slightly from the perfect sphericity and corresponds rather to an ellipsoid with an aspect ratio of $1.10 \pm 0.05$, both simulation methods return values which are closer to the experimental one $(2.22 \mathrm{eV})$ : $2.27 \mathrm{eV}$ (ADE-FDTD simulations) and $2.35 \mathrm{eV}$ (MNPBEM simulations).

A part of this discrepancy between simulations and experiments could be attributed to the fact that, for the simulations, the dielectric function for the Au NP is approximated to its bulk value. However, it has been shown elsewhere $[3,115]$ that an incorporation of nonlocal size effects into the dielectric function is not necessary for the calculation of the optical properties of nanometer-sized spheres as long as the diameter of the spheres is above $8 \mathrm{~nm}$. However, a similar value $(2.27 \mathrm{eV})$ for $20-\mathrm{nm}-$ diameter gold nanospheres in an $n=1.5$ medium has already been reported by another group [116], who measured this value by optical means and calculated it using Mie theory [117], and the dielectric function of gold reported by Johnson and Christy [118]. (See also Sec. V B 2 for a discussion of the discrepancy between the experimental value and the analytical model.)

The rest of this discrepancy between simulations and experiments is (from the experimental point of view) probably due to fabrication artifacts (like surface roughness and grain boundaries), which could affect the dielectric function of the Au NPs, as well as (from the numerical 
point of view) to the utilization of a tabulated dielectric function from the literature [111] (and thus not measured on our NPs), and to the fitting of this function by Drude and Lorentz oscillators which results in small deviations from the tabulated values (see Sec. II C in the Supplemental Material [78]).

The discrepancy between the two simulation methods [2.27 eV (ADE-FDTD simulations) and $2.35 \mathrm{eV}$ (MNPBEM simulations)] is expected (see Sec. III), even if both methods use the same dielectric function of gold as input. We believe this discrepancy to be due to (a) different definitions of resonance (in the ADE-FDTD simulations, the energy at which the total normalized electric field $|\mathbf{E}| /\left|\mathbf{E}_{0}\right|$ is maximized in an arbitrary point of the specimen is taken as the resonance energy, whereas, in the MNPBEM simulations, the energy at which the plasmonic excitation probability is maximized in an arbitrary point of the specimen is taken as the resonance energy), (b) different numerical solution algorithms of the Maxwell equations employed by the two methods, and (c) the existence of different geometrical modeling inaccuracies between the two methods (the ADE-FDTD method discretizes the volume of the nanoparticle within the staircase approximation, whereas the MNPBEM method discretizes the surface of the nanoparticle through a surface triangulation).

\section{Prolate faceted nanoparticle}

The simulation results for the prolate faceted nanoparticle are in good agreement with those obtained from EELS analysis and the observed shifts can be explained by the morphological differences between the real and the simulated NP.

Both the ADE-FDTD simulations (1.78 and $2.29 \mathrm{eV}$ ) and the MNPBEM simulations (1.94 and $2.31 \mathrm{eV}$ ) return energy values for the longitudinal and the transverse plasmon resonance energies which are close to the experimental ones (1.90 and $2.33 \mathrm{eV}$ ). The discrepancies are most probably due to morphological differences between the simulated NP and the real NP, which most probably is not a perfect octahedron with rounded corners and edges but instead can have a slightly irregular shape which can be revealed only by electron tomography experiments.

As in the case of the nanosphere, we believe the discrepancy between the two simulation methods $[1.78 \mathrm{eV}$ (ADE-FDTD simulations) and $1.90 \mathrm{eV}$ (MNPBEM simulations)] to be due to (a) different definitions of resonance, (b) different numerical solution algorithms, and (c) different geometrical modeling inaccuracies.

\section{Nanorod}

The simulation results for the nanorod are in good agreement with those obtained from EELS analysis. Both the ADE-FDTD simulations (1.24 and $1.91 \mathrm{eV}$ ) and the MNPBEM simulations (1.29 and $1.95 \mathrm{eV})$ return energy values for the longitudinal and the transverse plasmon resonance energies which are close to the experimental ones (1.18 and $1.80 \mathrm{eV})$. The discrepancies can be attributed to the different dimensions of the nanoparticle and to the probable slightly irregular shape of the real nanoparticle, as well as (as discussed in the case of the nanosphere) to likely differences between the actual dielectric function of the NP and to the one which is used for numerical simulations.

As in the case of the nanosphere and the prolate faceted nanoparticle, we believe the discrepancy between the two simulation methods [1.24 and $1.91 \mathrm{eV}$ (ADE-FDTD simulations) and 1.29 and $1.95 \mathrm{eV}$ (MNPBEM simulations)] to be due to (a) different definitions of resonance, (b) different numerical solution algorithms, and (c) different geometrical modeling inaccuracies.

\section{Nanowire}

Again, the simulation results for the nanowire are in good agreement with those obtained from EELS analysis. Both the ADE-FDTD simulations $(0.67,1.15$, and $1.46 \mathrm{eV})$ and the MNPBEM simulations $(0.67,1.17$, and $1.53 \mathrm{eV})$ return energy values for the longitudinal and transverse plasmon resonance energies which are close to the experimental ones $(0.86,1.27$, and $1.66 \mathrm{eV})$. The discrepancies can be attributed to the different dimensions of the nanoparticle and to the probable slightly irregular shape of the real nanoparticle. In particular, the HAADF image suggests that the nanowire is most probably not a perfect cylinder but is gradually thinned down at its ends. Retardation effects which should be noticeable for nanoparticles with dimensions approaching that of the wavelength of exciting light in the surrounding matrix can most probably be excluded as a possible cause of the discrepancies between the experiments and the numerical simulations, as they are accounted for in both the ADE-FDTD and MNPBEM simulations. However, as the diameter of the nanowire is $7.8 \pm 1.3 \mathrm{~nm}$, which is comparable to the approximate limit of $8 \mathrm{~nm}$ reported elsewhere [3,115], an incorporation of nonlocal size effects into the dielectric function might be necessary for the calculation of plasmonic resonance energies for such a thin nanowire.

As in the case of the previous three nanoparticles, we believe the slight discrepancy between the two simulation methods [0.67, 1.15, and $1.46 \mathrm{eV}$ (ADE-FDTD simulations) and $0.67,1.17$, and $1.53 \mathrm{eV}$ (MNPBEM simulations)] to be due to (a) different definitions of resonance, (b) different numerical solution algorithms, and (c) different geometrical modeling inaccuracies.

\section{EXPERIMENTAL AND ANALYTICAL PLASMONIC DISPERSION RELATION}

\section{A. Experimental (STEM-EELS) dispersion relation}

In order to compare our experimental data to analytical models, we determine the dispersion relation of the 


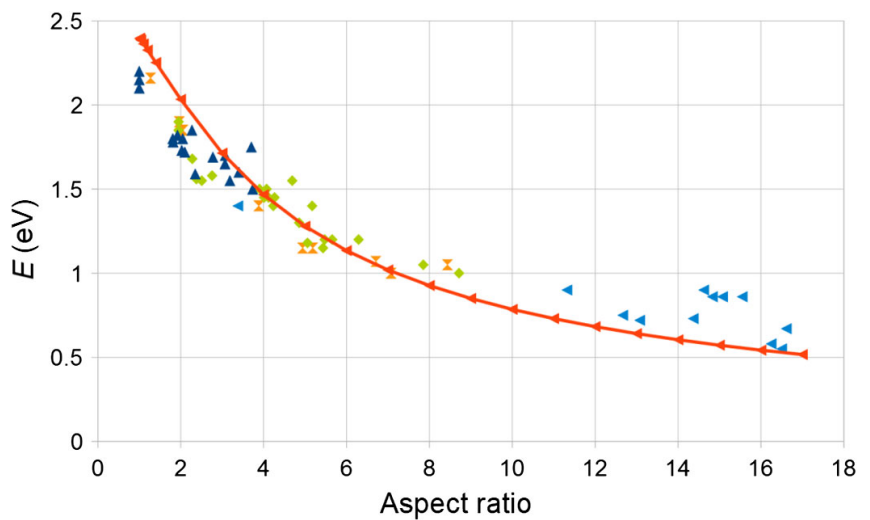

FIG. 7. Experimental (STEM-EELS) dipolar plasmonic mode energies of all 66 studied nanoparticles versus their aspect ratio $\mathcal{A}=L / w$ (where $L$ is the length and $w$ the width of the nanoparticle). Different length ranges are coded with different colors and symbols [dark blue (triangles pointing upwards), $L<30 \mathrm{~nm}$; green (squares), $30 \mathrm{~nm}<L<60 \mathrm{~nm}$; orange (vertical bow ties), $60 \mathrm{~nm}<L<90 \mathrm{~nm}$; light blue (triangles pointing to the left), $90 \mathrm{~nm}<L]$. The orange line indicates predictions (without fitting) based on the quasistatic model (see Sec. V B 2)
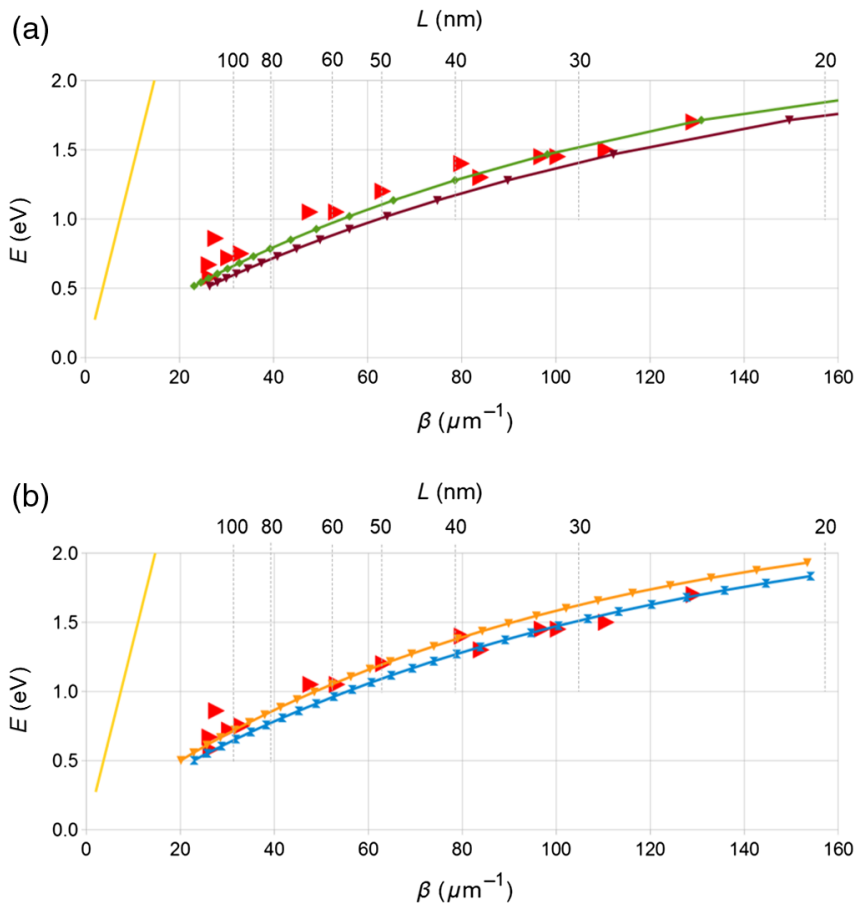

FIG. 8. Experimental (STEM-EELS) dipolar plasmonic mode energies of the nanoparticles of width $w$ between 7.0 and $8.0 \mathrm{~nm}$ versus the wave vector $\beta=(m \pi / L)$ of the plasmonic excitation, with $m=1$ for the dipolar mode. (a) Burgundy line and green line, predictions (without fitting) based on the quasistatic model (see Sec. V B 3) for nanoellipsoids of different widths [burgundy line (lower line), $7 \mathrm{~nm}$; green line (upper line), $8 \mathrm{~nm}$ ]. (b) Blue line and orange line, predictions (without fitting) based on the waveguide model (see Sec. V B 3) for nanowires of different widths [blue line (lower line), $7 \mathrm{~nm}$; orange line (upper line), $8 \mathrm{~nm}$ ]. Yellow line (line in the upper left corner), light line in $\mathrm{SiO}_{2}$ (see Sec. II C in the Supplemental Material [78] for parameters). plasmonic excitations in the nanoparticles studied here from the collected experimental STEM-EELS data (as was also done by other groups for other NPs $[46,102,119,120])$.

For this purpose, all of the experimental STEM-EELS results of a series of experiments including nanospheres, prolate faceted nanoparticles, nanorods, and nanowires are regrouped in Figs. 7 and 8.

Figure 7 shows the dipolar plasmonic mode energies of all 66 studied nanoparticles versus their aspect ratio $\mathcal{A}=$ $L / w$ (where $L$ is the length and $w$ the width of the nanoparticle). The results of different length ranges of the nanoparticles $(L<30 \mathrm{~nm}, 30 \mathrm{~nm}<L<60 \mathrm{~nm}$, $60 \mathrm{~nm}<L<90 \mathrm{~nm}$, and $90 \mathrm{~nm}<L$ ) are coded with different colors (dark blue, green, orange, and light blue) in Fig. 7. The widths of the nonirradiated nanospheres (7 out of the 66 NPs, including the nanosphere shown in Fig. 3) equal $23 \pm 1.5 \mathrm{~nm}$. The widths of 53 irradiated NPs (including the nanorod shown in Fig. 5, and the nanowire shown in Fig. 6) range from 5.4 to $16.5 \mathrm{~nm}$, with an average value of $9.5 \mathrm{~nm}$ and a standard deviation of $2.9 \mathrm{~nm}$. The remaining six irradiated NPs (including the faceted nanoparticle shown in Fig. 4) develop from particularly large nanospheres and have particularly large widths ranging from 26.4 to $55 \mathrm{~nm}$ (average value of $33.3 \mathrm{~nm}$ and standard deviation of $10.9 \mathrm{~nm})$. The lengths of the nonirradiated nanospheres ( 7 out of the 66 NPs, including the nanosphere shown in Fig. 3) equal $23 \pm 1.5 \mathrm{~nm}$. The lengths of 57 irradiated NPs (including the faceted nanoparticle shown in Fig. 4, the nanorod shown in Fig. 5, and the nanowire shown in Fig. 6) range from 23.5 to $120 \mathrm{~nm}$, with an average value of $53 \mathrm{~nm}$ and a standard deviation of $30 \mathrm{~nm}$. The remaining two irradiated NPs develop from particularly small nanospheres and have particularly short lengths $(18.4$ and $20 \mathrm{~nm})$. Analyzing Fig. 7, one notices that, with an increasing aspect ratio, the energy of the dipolar mode is redshifted, which is consistent with theory and previous work [121-123].

Figure 8 shows only the dipolar plasmonic mode energies of the nanoparticles of width $w$ between 7.0 and $8.0 \mathrm{~nm}$ versus the wave vector $\beta=(m \pi / L)$ of the plasmonic excitation, with $m=1$ for the dipolar mode, which implies a phase shift of zero upon reflection at the interfaces (see Sec. III of the Supplemental Material [78] and Refs. $[46,124,125]$ for a more detailed discussion). The lengths of these nanoparticles range from 24 to $120 \mathrm{~nm}$. Analyzing Fig. 8, one notices that the dispersion plot is consistent with that of a propagating surface-plasmonpolariton wave [2], i.e., close to the light line in $\mathrm{SiO}_{2}$ for small $\beta$ values and consistent with a saturation at high $\beta$.

In this type of representation, it is not possible to show all of our experimental values in a single graph because of the different widths of the NPs we study. (Even in an energy versus $\beta w$ representation it would not be possible to show all of our experimental values in a single graph as, in theory, the plasmonic mode energies $E$ are expected to depend not only 
on the quantity $\beta w$ but also, explicitly, on the NP diameter $w$ as soon as retardation effects are taken into account.) Thus, a width interval has to be chosen for this representation. The reason for choosing a width interval between 7.0 and $8.0 \mathrm{~nm}$ is that it contains a sufficient number of experimental values from both long and short prolate NPs.

The determination of the wave vector $\beta$ of the plasmonic excitation from the plasmonic resonance energy and the nanoparticle dimensions (as done in Sec. VA for Fig. 8) requires knowledge of the phase shift the plasmon is subjected to when reflecting from one of the interfaces between the metallic nanoparticle and the surrounding dielectric matrix. In this work, the phase shift upon reflection at the $\mathrm{Au} / \mathrm{SiO}_{2}$ interface is considered to be equal to 0 (see, however, other work $[46,124,125]$ for a further discussion of this subject), which is acceptable here, as the resulting imprecision (approximately 20\%) is comparable to the one coming from the determination of aspect ratios of the nanoparticles (approximately 10\%; see Sec. III of the Supplemental Material [78] for a more detailed discussion).

Longitudinal plasmon redshifts and transverse plasmon blueshifts and the apparition of higher longitudinal plasmon modes when the nanoparticle sizes and aspect ratios increase have already been predicted [126] and experimentally demonstrated in the literature [46-48]. Figures 7 and 8 demonstrate that the longitudinal resonance frequencies of the Au nanoparticles in a silica matrix are tunable to a high degree by adjusting their lengths and diameters.

\section{B. Comparison with analytical dispersion relations}

Even if numerical MNPBEM and ADE-FDTD simulations can simulate the experimental results with a satisfactory precision and are the only way of predicting the energies of less regular realistic nanoparticle shapes, it is analytical models that especially provide physical insight into the plasmonic excitations of metallic nanoparticles.

Analytical models allow one to formulate hypotheses on how the plasmon energies are expected to depend on the size and shape of the nanoparticles. Comparing analytical models against the experimental results allows one to check experimentally the validity regions of the approximations which underly the analytical model used.

In this section, we introduce two complementary analytical models, the quasistatic model (expected to simulate small-aspect-ratio nanoparticles well) and the waveguide model (expected to simulate high-aspect-ratio nanoparticles well). Comparing the experimental results from the previous section to the analytical results obtained from these two models allows us to determine above which nanowire length the plasmonic excitations in the nanowires studied here may be better approximated by the infinite nanowire approximation (i.e., the waveguide model), rather than the high-aspectratio ellipsoid approximation (i.e., the quasistatic model).

\section{Analytical models (quasistatic model and waveguide model)}

The quasistatic model [3] can be used to estimate the energy of the dipolar plasmon resonance in nanoparticles which can be approximated by an ellipsoid. It is based on electrostatic theory with an additional assumption: the dimensions of the nanoparticle are considered to be much smaller than the wavelength of the incident light (respectively the wavelength of the photon having the same energy as the plasmon mode of the nanoparticle). As a consequence, the nanoparticle behaves like an ideal dipole in the oscillating field of incident light or in the oscillating field caused by an incident electron from the electron beam of the STEM. The analytical development of this model is retraced in Sec. IVA of the Supplemental Material [78]. Additional details can be found in Refs. [3,127].

The waveguide model can be used to estimate the energies of plasmon resonances in nanoparticles, which can be approximated by long cylinders with high aspect ratios like nanorods and nanowires. In this model, plasmonic excitations are interpreted as standing waves created by electromagnetic modes propagating in metal nanostructures with rotational symmetry surrounded by a dielectric material and reflecting at the ends of the nanostructure. The analytical development of this model is retraced in Sec. IV B of the Supplemental Material [78]. Additional details can be found in Refs. [128-130].

\section{Analytical dispersion relation (energy vs aspect ratio)}

The analytical "energy vs aspect ratio" dispersion relation can be calculated with the quasistatic model. As within this model the plasmonic resonance energy depends only on the aspect ratio of the nanoparticle and not explicitly on its length and its width, the experimental data of nanoparticles of different widths can be compared to the same analytical curve at the same time.

Figure 7 compares the results of the quasistatic model (without fitting) to the experimental data. From the point of view of the NP length, one can see that the plasmonic excitation energies of the NPs which are shorter than $90 \mathrm{~nm}$ (the dark blue, green, and orange symbols) are (as expected) well predicted by the quasistatic model. However, exceptions are the NPs shorter than $30 \mathrm{~nm}$, which are slightly overestimated by the quasistatic model. The plasmonic excitation energies of the nanowires which are longer than $90 \mathrm{~nm}$ (or,

TABLE II. Analytical energy vs aspect-ratio dispersion relation (quasistatic model) compared to the experimental energy values.

\begin{tabular}{lccc}
\hline \hline Aspect ratio & $1<\mathcal{A}<3.5 \pm 0.5$ & $3.5 \pm 0.5<\mathcal{A}<10 \pm 2$ & $10 \pm 2<\mathcal{A}<16 \pm 1$ \\
\hline$\Delta E_{\mathrm{QM}}(\mathcal{A})$ & $-0.2 \pm 0.2 \mathrm{eV}$ & $0.0 \pm 0.2 \mathrm{eV}$ & $0.2 \pm 0.2 \mathrm{eV}$ \\
\hline \hline
\end{tabular}


rather, longer than $100 \pm 10 \mathrm{~nm}$, upon closer inspection of the experimental values; the light-blue symbols) are (as expected) underestimated by the quasistatic model, which is supposed to describe only those NPs with small aspect ratios. In addition, the quasistatic model does not take into account any retardation effects which could become non-negligible for higher-aspect-ratio NPs.

From the point of view of the aspect ratio of the nanoparticle, Table II summarizes the observations from Fig. 7. $\Delta E_{\mathrm{QM}}(\mathcal{A})$ is defined as $E_{\text {expt. }}(\mathcal{A})-E_{\mathrm{QM}}(\mathcal{A})$, with $\mathcal{A}$ being the aspect ratio. The value of $\Delta E_{\mathrm{QM}}(\mathcal{A})$ is $-0.2 \pm 0.2 \mathrm{eV}$ for $1<\mathcal{A}<3.5 \pm 0.5, \quad 0.0 \pm 0.2 \mathrm{eV}$ for $3.5 \pm 0.5<\mathcal{A}<$ $10 \pm 2$ and $0.2 \pm 0.2 \mathrm{eV}$ for $10 \pm 2<\mathcal{A}<16 \pm 1$ As said above, a nonzero $\Delta E_{\mathrm{QM}}(\mathcal{A})$ is expected for $10 \pm 2<\mathcal{A}<16 \pm 1$, but it might seem surprising for $1<\mathcal{A}<$ $3.5 \pm 0.5$.

Based on these data, one possible interpretation could be that, as the dielectric polarizability of a cylinder is close to that of an ellipsoid [131], the quasistatic model works well for medium aspect ratios $(3.5 \pm 0.5<\mathcal{A}<10 \pm 2)$ until retardation effects can no longer be neglected for higher $\mathcal{A}$ values $(10 \pm 2<\mathcal{A})$, but that it does not work well for small aspect ratios $(1<\mathcal{A}<3.5 \pm 0.5)$ because the nanoparticles of these aspect ratios are neither ellipsoids nor cylinders but rather are octahedrons.

Nonetheless, it might still seem surprising that the nanospheres studied here with an aspect ratio of 1 are not well described by the quasistatic model: Fig. 7 shows that the experimental values for the ( $23 \pm 1.5)$-nm-diameter nanospheres are overestimated by between 0.2 and $0.3 \mathrm{eV}$. A possible explanation for this redshift of the experimental value with respect to the analytical value for a perfect sphere [as well as for the small-aspect-ratio $(1<\mathcal{A}<$ $3.5 \pm 0.5)$ nanoparticles described above] could be that, due to the low penetration depth of the electric field in gold (which is between 12 and $19 \mathrm{~nm}$ in the near-infrared and visible range between 1.5 and $2.4 \mathrm{eV}$ in which the quasistatic approximation overestimates the experimental values, as seen in Fig. 7), the electric field (even though it penetrates the nanoparticle) is not homogeneous inside the nanoparticle, and thus the quasistatic approximation (which assumes a homogeneous electric-field distribution inside the nanoparticle) is not sufficiently precise for nanoparticles of diameters higher than $20 \mathrm{~nm}$, which is the case for all of the nanospheres studied here, as well as for many small-aspect-ratio $(1<\mathcal{A}<3.5 \pm 0.5)$ nanoparticles [see, e.g., the prolate faceted nanoparticle in Fig. 4(a)].

Finally, a slight nonsphericity of the NP in the direction of the electron beam could explain the rest of the redshift of the experimental value with respect to the analytical value for a perfect sphere seen in Fig. 7. Even if ion irradiation breaks the isotropy of the system by defining a privileged direction (the direction of the ion beam) in which the NPs will elongate and, as a consequence, all irradiated NPs are elongated perpendicular to the electron beam, this argument does not apply to the nanospheres studied here, which (as they have not been irradiated) can be elongated in any arbitrary direction like, also, the direction of the electron beam, in which case their nonsphericity remains undetected.

In conclusion, based on a study of 66 nanoparticles with widths between 5.4 and $55 \mathrm{~nm}$ and lengths between 18.4 and $120 \mathrm{~nm}$, the quasistatic model predicts the dipolar plasmonic resonance energies with a $\Delta E$ of $-0.2 \pm 0.2 \mathrm{eV}$ for $1<\mathcal{A}<3.5 \pm 0.5,0.0 \pm 0.2 \mathrm{eV}$ for $3.5 \pm 0.5<\mathcal{A}<$ $10 \pm 2$ and $0.2 \pm 0.2 \mathrm{eV}$ for $10 \pm 2<\mathcal{A}<16 \pm 1$, with $\mathcal{A}$ being the aspect ratio of the NP. The small deviations might be due to the octahedral NP shapes and to overly large NP widths (compared to the penetration depths) at small $\mathcal{A}$ and to retardation effects at large $\mathcal{A}$.

\section{Analytical dispersion relation (energy vs wave vector)}

The analytical "energy vs wave vector" dispersion relation can be calculated with the quasistatic model or (independently) with the waveguide model. However, as in this representation the wave vector $\beta$ depends explicitly on the length $L$ of the NP, different analytical curves are expected for NPs of different widths (according to the quasistatic model) because two NPs of the same length but different widths have a different aspect ratio and thus a different resonance energy. The waveguide model which approximates the NPs as long cylindric waveguides also predicts different analytical curves for waveguides of different diameters (i.e., widths). As a consequence, it is not possible to compare all of the experimental values from Fig. 7 to the same analytical curve (see also Sec. VA). A width interval thus has to be chosen. The reason for choosing the width interval between 7.0 and $8.0 \mathrm{~nm}$ is that it contains a sufficient number of experimental values from both long and short prolate NPs.

Figure 8(a) compares the results of the quasistatic model (drawn for a nanowire width of $7 \mathrm{~nm}$ and for a nanowire width of $8 \mathrm{~nm}$, and without fitting) to the experimental data of nanowires of widths between 7 and $8 \mathrm{~nm}$. Figure $8(\mathrm{~b})$ does the same for the results of the waveguide model (drawn for a nanowire width of $7 \mathrm{~nm}$ and for a nanowire width of $8 \mathrm{~nm}$, and without fitting).

Table III summarizes the observations from Fig. 8. $\Delta E_{\mathrm{QM}}(\beta)$ is defined as $E_{\text {expt. }}(\beta)-E_{\mathrm{QM}}(\beta)$, with $E_{\mathrm{QM}}(\beta)$ being defined as $\left[E_{\mathrm{QM}}(7 \mathrm{~nm})(\beta)+E_{\mathrm{QM}}(8 \mathrm{~nm})(\beta)\right] / 2$. $\Delta E_{\mathrm{WM}}(\beta)$ is defined analogously.

TABLE III. Analytical energy vs wave-vector dispersion relations (quasistatic model and waveguide model) compared to the experimental energy values.

\begin{tabular}{lrr}
\hline \hline$L$ & $L<40 \pm 5 \mathrm{~nm}$ & $40 \pm 5 \mathrm{~nm}<L$ \\
\hline$\Delta E_{\mathrm{QM}}(\beta)$ & $0.05 \pm 0.05 \mathrm{eV}$ & $0.15 \pm 0.05 \mathrm{eV}$ \\
$\Delta E_{\mathrm{WM}}(\beta)$ & $-0.05 \pm 0.05 \mathrm{eV}$ & $0.05 \pm 0.05 \mathrm{eV}$ \\
\hline \hline
\end{tabular}


As for the quasistatic model [see Fig. 8(a) and Table III], it predicts the plasmonic excitation energies of the nanowires very well for $\beta>70 \pm 10 \mu \mathrm{m}^{-1}$ (i.e., for $L<45 \pm 5 \mathrm{~nm}$ ). The symbols lie between the two solid lines, and $\Delta E=0.05 \pm 0.05 \mathrm{eV}$. The same model slightly underestimates (as expected) the plasmonic excitation energies of the nanowires above this nanowire length (i.e., for $L>45 \pm 5 \mathrm{~nm}$ ), where $\Delta E=0.15 \pm 0.05 \mathrm{eV}$.

The reason for the difference of the limit values obtained from Fig. $7(100 \pm 10 \mathrm{~nm})$ and Fig. 8 (a) $(45 \pm 5 \mathrm{~nm})$ is that, in Fig. 7, we have a wide distribution of values and therefore do not see a difference between the experimental data and the quasistatic model until it is approximately $0.2 \mathrm{eV}$. By contrast, Fig. 8(a) features a very narrow distribution of experimental values, which already makes a smaller difference of approximately $0.1 \mathrm{eV}$ of the experimental data and the quasistatic model apparent. It is obvious that a precise limit value does not exist but is dependent on the precision with which one wants to predict the plasmonic excitation energies.

As for the waveguide model [see Fig. 8(b) and Table III], one notices that the analytical curves for 7- and 8-nmdiameter cylinders lie slightly higher than the corresponding analytical curves of the quasistatic model for 7- and 8-nmwide ellipsoids in Fig. 8(a), and that therefore (in contrast to the quasistatic model) the waveguide model allows one to predict the experimental values with a small imprecision over the entire studied $\beta$ range: $\Delta E=-0.05 \pm 0.05 \mathrm{eV}$ for $\beta>$ $70 \pm 10 \mu \mathrm{m}^{-1}$ (i.e., for $L<45 \pm 5 \mathrm{~nm}$ ) and $\Delta E=0.05 \pm$ $0.05 \mathrm{eV}$ for $\beta<70 \pm 10 \mu \mathrm{m}^{-1}$ (i.e., for $L>45 \pm 5 \mathrm{~nm}$ ).

The good agreement between the waveguide model and the experimental data is particularly interesting for high $\beta$ values (i.e., short NPs), as one would not expect the waveguide model to perform this well for this kind of small aspect ratio NPs: the two rightmost NPs in Fig. 8 have aspect ratios of 3.1 and 3.7, respectively). From another point of view, the aspect ratios above of 3.1 and 3.7 are (even if small) already sufficiently high for the NPs to assume a sufficiently cylindrical shape (as opposed to an octahedral shape for lower aspect ratios of roughly $2 \pm 0.5$ ), so the quasistatic model makes accurate predictions as well.

However, surprisingly, the plasmonic resonance energy of one nanowire situated at $\beta<30 \mu \mathrm{m}^{-1}$ (i.e., $L>100 \mathrm{~nm}$ ) [see Fig. 8(b)] is underestimated by the waveguide model by between 0.2 and $0.3 \mathrm{eV}$. (The same energy is underestimated by the quasistatic model by approximately $0.3 \mathrm{eV}$ as well, but while the quasistatic model is expected to work less well for longer NPs, the waveguide model is supposed to work well for longer NPs as well.) The most probable reason are experimental inaccuracies like a probable inaccurate determination of the width or energy of this nanowire. This hypothesis is supported by the fact that four other nanowires of similar lengths [i.e., $L \geq 100 \mathrm{~nm}$; see Fig. 8(b)] are well estimated by the waveguide model.
It should also be pointed out that the quasistatic model naturally takes into account the phase shift $\phi$ upon reflection at the interfaces; thus, the arguments developed in Sec. III of the Supplemental Material [78] do not apply to the quasistatic model. This means that if one corrects the data shown in Fig. 8(a) with the correct value of $\phi$, only the $\beta$ axis has to be rescaled. The experimental data and the modeling curve in Fig. 8(a) would not shift in respect to each other.

By contrast, the arguments developed in Sec. III of the Supplemental Material [78] fully apply to the waveguide model and the aspect ratios [and thus also the experimental $\beta$ values in Fig. 8(b)] might have a relative error of up to $20 \%$, which, upon correction with the correct value of $\phi$, would shift the experimental values in respect to the modeling curve in Fig. 8(b).

In conclusion, based on a study of 14 nanoparticles of widths between 7 and $8 \mathrm{~nm}$ and lengths between 24 and $120 \mathrm{~nm}$, the quasistatic model (which approximates the NPs as ellipsoids and which is thus expected to work better for low-aspect-ratio NPs) predicts the plasmonic excitation energies of nanowires with lengths $25 \pm 5 \mathrm{~nm}<L<45 \pm$ $5 \mathrm{~nm}$ within a precision of $\Delta E=0.05 \pm 0.05 \mathrm{eV}$ and underestimates (as expected) those of the longer nanowires $(45 \pm 5 \mathrm{~nm}<L<110 \pm 10 \mathrm{~nm})$, with $\Delta E=0.15 \pm 0.05 \mathrm{eV}$. By contrast, the waveguide model (which approximates the NPs as long cylindric waveguides and which is thus expected to work better for high-aspect-ratio NPs) allows one to predict the experimental values with a small imprecision over the entire studied nanowire length $L$ range: $\Delta E=-0.05 \pm 0.05 \mathrm{eV}$ for $25 \pm 5 \mathrm{~nm}<L<45 \pm 5 \mathrm{~nm}$ and $\Delta E=0.05 \pm 0.05 \mathrm{eV}$ for $45 \pm 5 \mathrm{~nm}<L<110 \pm 10 \mathrm{~nm}$. However, one NP situated at $\beta<30 \mu \mathrm{m}^{-1}$ (i.e., $L>100 \mathrm{~nm}$ ) could not be correctly predicted, most probably, due to experimental inaccuracies.

\section{APPLICATIONS}

\section{A. The different types of ion-beam-shaped nanoparticle arrays (IBSNAs)}

Possible applications of IBSNAs are abundant and can be sorted into applications of "widely spaced" IBSNAs (in which the nanoantennas behave like isolated nanoantennas) and of "narrowly spaced" IBSNAs (in which the main feature is the appearance of hybridized plasmonic modes) on one hand, and into applications of "surface array" IBSNAs (in which the nanoantennas are close to the surface of the $\mathrm{SiO}_{2}$ specimen) and of "volume array" IBSNAs (in which the nanoantennas are in the volume of the $\mathrm{SiO}_{2}$ specimen far from its surfaces) on the other hand.

In this section, wide spacing means that the nanoantennas are far enough apart so that hybridized plasmonic modes do not appear. Nonetheless, even the wide spacing can be a very dense spacing with interantenna distances below the wavelength of the incident light [132], respectively the wavelength of the photon having the same energy 
as the plasmon mode of the nanoparticle. In particular, Figs. 5(i) and 6(k) show that the excitation probability decreases rapidly with the lateral distance to the particle (to $10 \%$ and $30 \%$, respectively, of the maximum excitation probability at a lateral distance on the order of $20 \mathrm{~nm}$ ). If one takes $10 \%$ of the maximum excitation probability as the cutoff criterion, Fig. 5(i) indicates that, for nanoparticles with intermediate aspect ratios $(5 \pm 1)$, even a spacing as small as $20 \mathrm{~nm}$ is already a wide spacing, whereas, for nanoparticles with high aspect ratios (12 \pm 3 ), an extrapolation of Fig. 6(k) indicates that a spacing of $45 \pm 5 \mathrm{~nm}$ is already a wide spacing (meaning that the nanoantennas behave as isolated nanoantennas).

In what follows, we first describe the possible applications of widely spaced IBSNAs (of both the surface-array type and the volume-array type) in more detail, then briefly comment on the possible applications of the corresponding narrowly spaced IBSNAs.

\section{B. IBSNAs for surface-enhanced Raman spectroscopy (SERS)}

As for what concerns the surface-array type of the widely spaced IBSNAs, a very interesting application is its utilization for SERS [133]. The main advantages are the easy tuning possibility of the resonance frequency through control of the nanoantenna aspect ratio, and also the possibility of enhancing the Raman signal within the infrared domain.

Figure 9 illustrates the fabrication procedure and the utilization of a surface-array IBSNA for SERS. First, a horizontal Au nanosphere array in $\mathrm{SiO}_{2}$ is irradiated by an oblique ion beam [see Fig. 9(a)]. Then, the $\mathrm{SiO}_{2}$ layer above the nanoantennas is removed by standard FIB methods or by HF etching [see Fig. 9(b)]. This modification of the dielectric environment of the nanoantennas is expected to induce a slight blueshift of the dipolar resonance of the nanoantennas which has to be compensated for by a slightly higher aspect ratio of the nanoantennas (compared to nanoantennas embedded in the volume of a $\mathrm{SiO}_{2}$ layer). Finally, molecules deposited on the surface of the specimen [see Fig. 9(c)] can be detected with SERS in a confocal setup with a linearly polarized laser beam [see Fig. 9(d)].

The oblique orientation of the nanoantennas allows for a much better coupling between the long nanoantenna axis with the linearly polarized laser beam than in a vertical geometry (where the long nanoantenna axis would only have the possibility to couple to the weak longitudinal component of the linearly polarized laser beam). The field enhancement (and thus also the Raman signal emitted by the molecules) is stronger the closer the molecules are to the nanoantennas [see Fig. 9(d)]. In particular, Figs. 5(f) and $6(\mathrm{~g})$ show that not only can the electric-field enhancement $|\mathbf{E}| /\left|\mathbf{E}_{0}\right|$ reach values of approximately 50 in the immediate vicinity of the nanoantennas but that it still (a)

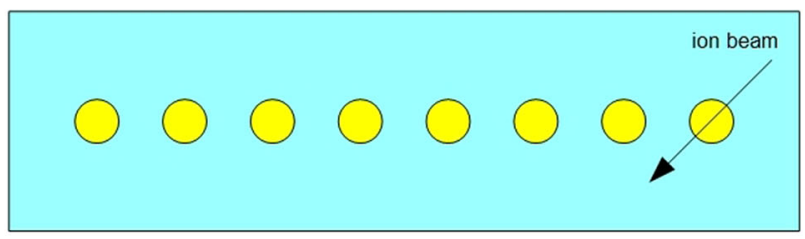

(b)

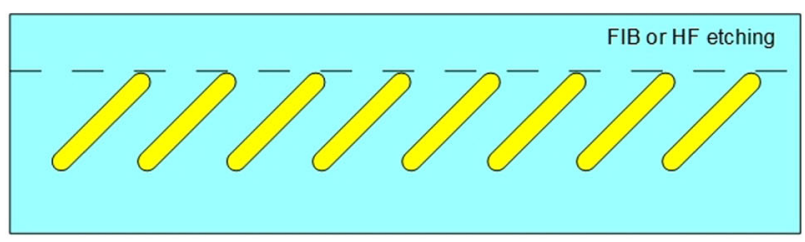

(c)

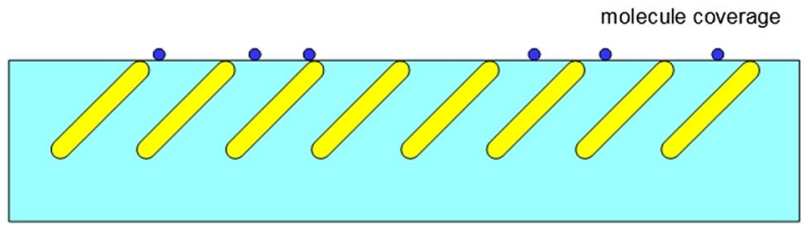

(d)

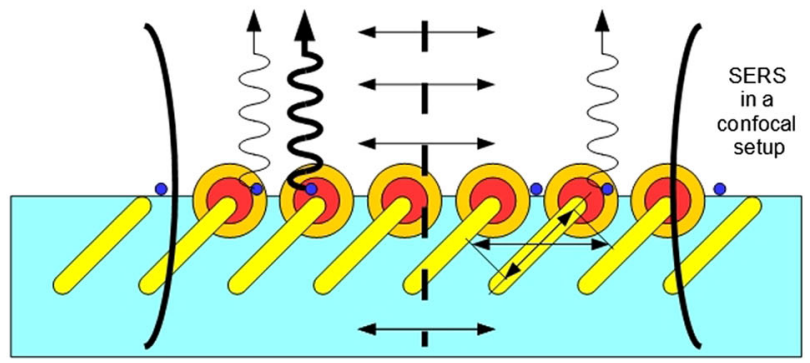

FIG. 9. Fabrication and utilization of a surface-array IBSNA for surface-enhanced Raman spectroscopy (SERS). (a) Irradiation of a horizontal Au nanosphere array in $\mathrm{SiO}_{2}$ by an oblique ion beam. (b) Removal of the $\mathrm{SiO}_{2}$ layer above the nanoantennas by FIB preparation or HF etching. (c) Molecule coverage. (d) Molecule detection with SERS in a confocal setup with a linearly polarized laser beam (not to scale as, for a 40-nm interantenna spacing, the laser beam would be at least ten spacings wide; see the text for further details).

reaches values of $7 \pm 2$ as far as $15 \mathrm{~nm}$ from the nanoantenna tip. As the SERS enhancement [133] equals $\left(|\mathbf{E}| /\left|\mathbf{E}_{0}\right|\right)^{4}$, this means that a two-dimensional nanoantenna lattice with a 40-nm spacing would enhance the SERS signal over $44 \%$ of the surface area of the specimen $\left[\pi \cdot(15 \mathrm{~nm})^{2} /(40 \mathrm{~nm})^{2}=0.44\right]$, with a factor of between $10^{3}$ and $10^{6}$.

In addition (if the spacing is above but on the same order of magnitude as the wavelength of the incident light), diffractive coupling between the ion-beam-shaped nanoparticles can (under the right incident conditions) lead to the creation of lattice-surface modes [134], which, in their spectral response of the IBSNA, manifest themselves as Fano resonances [134-136] and which can be tuned by the nanoantenna shape, orientation, and spacing [134, 137-139]. In contrast to the resonances of the individual nanoantennas, which usually feature quality factors smaller 
(a)
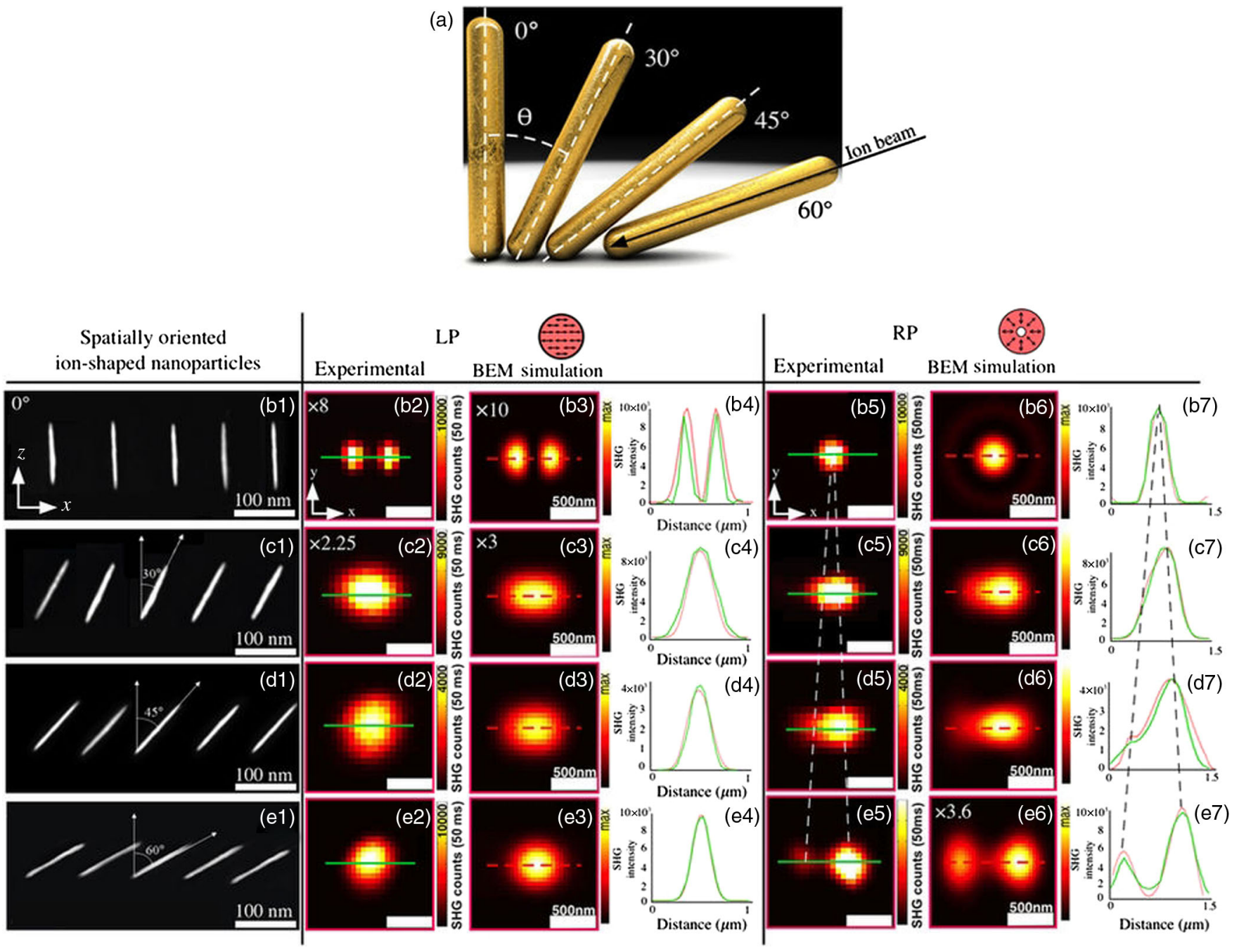

FIG. 10. Experimental and numerical second-harmonic images corresponding to oriented nanoantennas excited using focused linear and radial polarizations. (a) The four angular NP orientations, $\left(0^{\circ}, 30^{\circ}, 45^{\circ}\right.$, and $\left.60^{\circ}\right)$ are shown in columns (b1)-(e1). The experimental SHG intensity distributions are shown for different nanoantenna orientations using focused $x$-polarized linear polarization [LP; columns (b2)-(e2)] and radial polarization [RP; columns (b5)-(e5)]. The SHG intensity is always normalized to the highest intensity observed among the two polarizations, allowing a visual comparison. The experimental (green lines) and calculated (red lines) intensity profiles for the different nanoantenna orientations using both focused $x$-polarized linear and radial polarizations are also shown. [Columns (b3)(e3) and (b6)-(e6) are calculated SHG images using focused linear $(x)$ and radial polarizations under the same experimental conditions.] This figure was reproduced from Slablab et al. [140] under the Creative Commons CC BY license [141] and was slightly modified.

than 10, the quality factors of Fano resonances can reach several hundred $[134,138,139]$, which can be exploited for very selective molecule detection in SERS applications.

\section{IBSNA-based second-harmonic generator (SHG) and polarization-state analyzer of laser beams}

As for what concerns the volume-array type of the widely spaced IBSNAs, a very interesting application is its utilization for SHG [140]. The main advantage is the dependence of its SHG emission on the angle between the nanoantenna axis and the incidence direction of the laser beam, as well as on its polarization state. This dependence has already been shown experimentally on one of our
IBSNAs [140] for linearly polarized (LP) and radially polarized (RP) beams by acquiring spatially resolved (with respect to the laser beam position) SHG intensity signal maps (see Fig. 10) and by analyzing the maximum SHG intensity which can be generated depending on the tilt angle between the incidence direction of the laser beam and the long nanoantenna axis (see Fig. 11).

The dependence of the SHG emission on the polarization state of the laser beam is illustrated in Fig. 10. The SHG signal map for a focused LP laser beam [with nanoantennas aligned parallel to the laser beam; see Fig. 10(b1)] in Fig. 10(b2) features two symmetric lobes, with a dark spot in the center which corresponds to the location of the nanoantenna. The two lobes are produced by the weak 

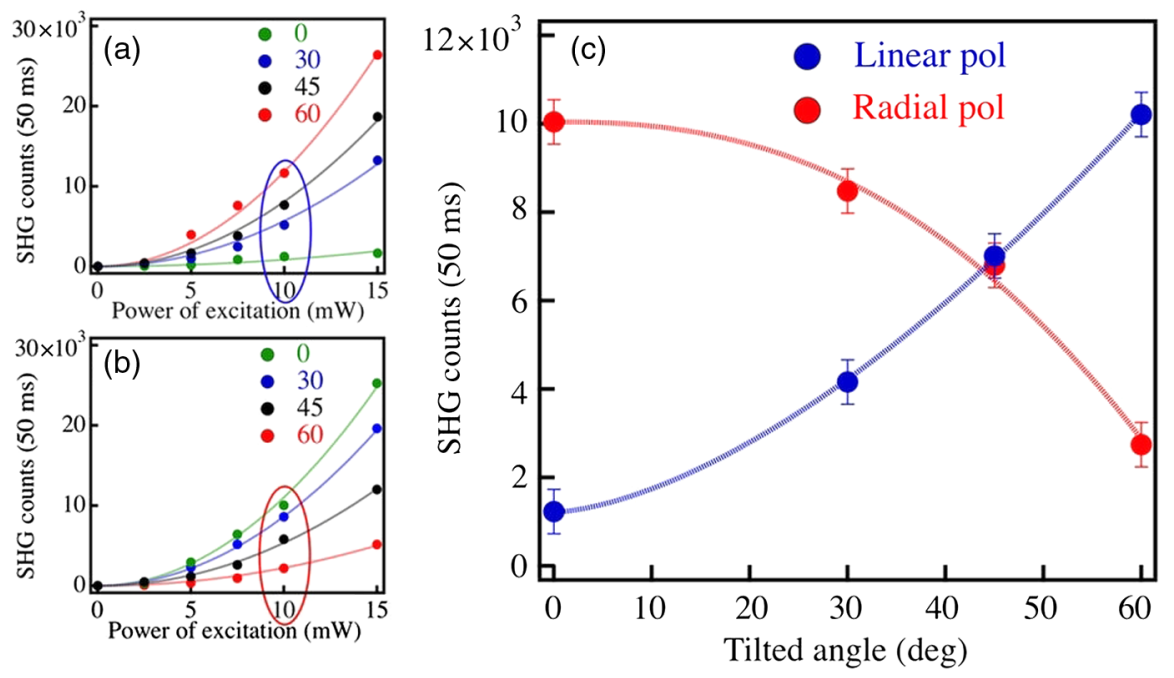

FIG. 11. (a)-(c) Experimental measurements of the maximum SHG signal from an isolated nanoantenna. (a) and (b) correspond to the quadratic dependence of the SHG signal on the laser input power for linear and radial polarizations, respectively, and for different nanoantenna orientations. (c) The maximum SHG signal as a function of the nanoantenna tilt angle for linear (blue) and radial (red) polarizations and for an average input power of $10 \mathrm{~mW}$. The dashed curves (red and blue) serve as guides for the eye. This figure was reproduced from Slablab et al. [140] under the Creative Commons CC BY license [141] and was slightly modified. longitudinal field components of the focused LP beam, which are offset from the geometrical focus in the direction of the incident linear polarization [142]. By contrast, the SHG signal maps for a focused RP laser beam (and the same nanoantenna orientation) in Fig. 10(b5) show a single strong spot centered at the location of the nanoantenna. The reason is that the longitudinal field of the RP laser beam is significantly stronger and is centered at the geometrical focus.

If the nanoantennas are tilted with respect to the incident laser beam [see Figs. 10(c1)-10(e1)], the intensity distribution changes drastically for both kinds of laser beams (LP and RP). In the case of the LP beam, the two symmetric lobes with a dark spot in the center merge into a single strong spot which corresponds to the center of the nanoantenna [see Figs. 10(c2)-10(e2)]. In the case of the RP beam, the single strong spot separates into two asymmetric lobes which correspond to the extremities of the nanoantenna [see Figs. 10(c5)-10(e5)].

In the case of the LP beam [see Figs. 10(c2)-10(e2)], the reason for the appearance of the single strong spot for tilted nanoantennas is the strong coupling of the linear in-plane field component of the LP laser beam to the long axis of the nanoantenna, which outweighs the coupling of the longitudinal field component to the long axis of the nanoantenna already for tilt angles as small as $30^{\circ}$. In the case of the RP beam [see Figs. 10(c5)-10(e5)], the reason for the appearance of the two lobes for tilted nanoantennas is the strong coupling of the radial in-plane field component of the RP laser beam to the long axis of the nanoantenna. If the RP laser beam is positioned in the center of the nanoantenna, the two opposed radial in-plane components cancel each other out, and only the weak SHG signal due to the weak coupling of the longitudinal field of the RP laser beam remains. The RP laser thus has to be positioned on one of the extremities of the nanoantenna (so that only one radial field component couples to the nanoantenna and not both) in order to maximize the SHG signal. The reason for the asymmetry of the lobes is the fact that, on one nanoantenna extremity, the contributions of the radial in-plane field component and of the longitudinal field component of the RP laser beam add up, whereas, on the other nanoantenna extremity, the contribution of the longitudinal field component attenuates the contribution of the radial in-plane field component of the RP laser beam.

The maximum SHG intensity which can be generated by an isolated nanoantenna is shown in Fig. 11. The dependence of this maximum SHG intensity on the tilt angle between the incident laser beam and the long axis of the nanoantenna shows a striking difference between the two studied polarization states. In the case of the LP laser beam, one maximizes the SHG signal by maximizing the tilt angle, whereas, in the case of the RP laser beam, one maximizes the SHG signal by minimizing the tilt angle. The reason is that, in the case of the LP beam, the strongest coupling to the long axis of the nanoantenna can be achieved through the linear in-plane field component of the LP laser beam (which requires a tilt angle as close as possible to $90^{\circ}$ ), whereas, in the case of the RP beam, the strongest coupling to the long axis of the nanoantenna can be achieved through the longitudinal field component of the RP laser beam (which requires a tilt angle as close as possible to $0^{\circ}$ ).

The sensitivity of the spatial SHG signal intensity distribution on the precise polarization state of the laser beam (as illustrated in Fig. 10) and the (precise polarization-state-dependent) sensitivity of the maximum SHG intensity generated by the nanoantenna on the tilt angle between the incident laser beam and the long axis of the nanoantenna (as illustrated in Fig. 11) can be exploited for the determination of the distribution of the incident electric field of a highly focused laser beam in its focal volume. The IBSNA can thus be used as a polarization-state analyzer of laser beams of unknown polarization states. 


\section{Applications of narrowly spaced IBSNAs}

Narrowly spaced IBSNAs (of both the surface-array type and the volume-array type) would be equally well suited as the corresponding widely spaced IBSNAs for the applications described above, with, however, the difference that (in contrast to the widely spaced IBSNAs) hybridized plasmonic modes would emerge. As these modes can only emerge if there is a non-negligible interaction between the plasmonic excitations on two adjacent nanoantennas, the narrowly spaced IBSNAs have to feature a sufficiently small interantenna spacing. As can be seen in Figs. 5(i) and $6(\mathrm{k})$, the excitation probability decreases rapidly with the lateral distance to the particle (to $10 \%$ and $30 \%$, respectively, of the maximum excitation probability at a lateral distance on the order of $20 \mathrm{~nm}$ ). This means that in order to generate hybridized plasmonic modes, one should use IBSNAs with high aspect ratios $(12 \pm 3)$ and very small interantenna distances $(<40 \mathrm{~nm})$.

However, because of the initial size of the nonirradiated $\mathrm{Au}$ nanospheres $(20 \mathrm{~nm})$ and the necessity to separate the nanospheres with a sufficiently thick layer of $\mathrm{SiO}_{2}$ (which we estimate to be $20 \mathrm{~nm}$ but which has yet to be confirmed by experiments) in order to prevent merging of the spheres and to ascertain the correct shaping of the nanoantennas, the minimum interantenna distance is limited in a conventional arrangement [see Figs. 12(a1) and 12(a2)] to values above $40 \mathrm{~nm}$ but could be reduced in an intertwined arrangement [see Figs. 12(b1) and 12(b2)] to values in the range of $20-40 \mathrm{~nm}$. Within this range, we expect the nanoantenna spacing to be an important tuning parameter of the plasmonic response of the IBSNA, and to enable a multitude of different plasmonic responses.
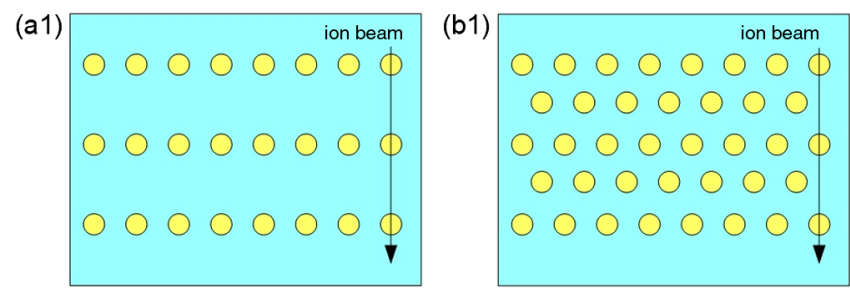

(a2)
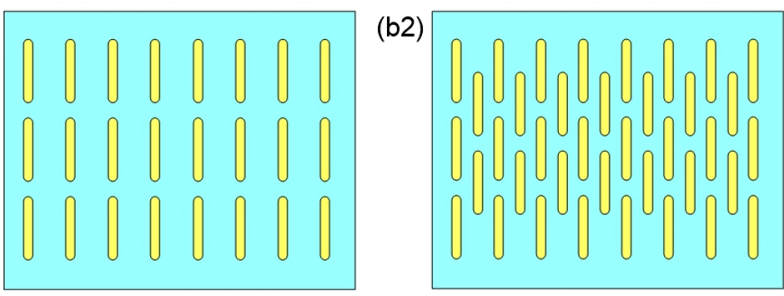

FIG. 12. Interantenna distance. (a) Smallest interantenna distance in an IBSNA achievable in a conventional arrangement [(a1) before ion-beam irradiation, (a2) after ion-beam irradiation]. (b) Smallest interantenna distance in an IBSNA achievable in an intertwined arrangement [(b1) before ion-beam irradiation, (b2) after ion-beam irradiation].
In particular, if the geometric parameters of the volume array type of the narrowly spaced IBSNAs are correctly chosen, we expect them to behave as hyperbolic metamaterials $[143,144]$. Hyperbolic metamaterials have possible applications in subwavelength resolution imaging, nanolithography, spontaneous emission enhancement, thermal coherent sources, and many other areas [143,144].

More generally, we would also expect the volume-array type of the narrowly spaced IBSNAs to also find their use in general wave-front shaping [132], as negative-index metamaterials [145], in superresolution imaging [17], and in invisibility cloaking [146].

To sum up, we expect ion-beam-shaped nanoparticle arrays to have an abundant number of interesting applications, above all in surface-enhanced Raman spectroscopy and second-harmonic generation, but also in conventional applications of metamaterials like negative refraction, superimaging, and invisibility cloaking.

\section{CONCLUSION}

In conclusion, we perform in this paper an experimental STEM-EELS nanoscale plasmon mapping study of how the energies and the shapes of the plasmonic resonances of spherical gold nanoparticles (fabricated by electron-beam lithography) evolve when the NP shape evolves from spheres to prolate faceted particles and cylinders, respectively (with the shape change being induced by swift heavy-ion irradiation), while their volume stays constant, and we compare these experimental results to numerical ADE-FDTD and MNPBEM simulations, as well as to two analytical models (the quasistatic model and the waveguide model). Four main results are obtained.

Our experimental STEM-EELS study allows us to directly confirm the evolution of the LSPRs in individual isolated prolate gold nanoparticles, nanorods, and nanowires as a function of the real shape of the nanoparticle in a symmetric environment with nanometric precision. First, these results confirm that the ion-beam-shaping process does not affect the plasmonic properties of the final particle in any negative way but instead produces nanoparticles whose plasmonic properties can be well predicted by theory. Second, these results confirm as well that the experimentally determined plasmonic properties of nanoparticles in a symmetric environment agree well with theory on a nanometric scale.

Third, these results confirm that the energies and shapes of dipolar plasmonic resonances, of higher-order longitudinal resonances, and of transverse resonances (for closeto-spherical NPs) are well predicted without fitting by the numerical ADE-FDTD and MNPBEM simulations within a precision of $0.1-0.2 \mathrm{eV}$. We expect to achieve better precision by a more precise modeling of the small deviations of the shape of the NPs from the perfect cylinder structure and by a more precise determination of the dielectric function by experimental means. 
Fourth, these results confirm that the two analytical quasistatic and waveguide models also allow one to estimate the dipolar mode energies of metallic NPs with similar precision but not for all nanoparticle lengths or aspect ratios. From the point of view of the nanoparticle aspect ratio and based on a study of 66 nanoparticles with widths between 5.4 and $55 \mathrm{~nm}$ and lengths between 18.4 and $120 \mathrm{~nm}$, the quasistatic model predicts dipolar plasmonic resonance energies with a $\Delta E$ of $-0.2 \pm 0.2 \mathrm{eV}$ for $1<\mathcal{A}<$ $3.5 \pm 0.5,0.0 \pm 0.2 \mathrm{eV}$ for $3.5 \pm 0.5<\mathcal{A}<10 \pm 2$, and $0.2 \pm 0.2 \mathrm{eV}$ for $10 \pm 2<\mathcal{A}<16 \pm 1$, with $\mathcal{A}$ being the aspect ratio of the NP. The small deviations might be due to the octahedral NP shapes and to overly large NP widths (compared to the penetration depths) at small $\mathcal{A}$ and to retardation effects at large $\mathcal{A}$. From the point of view of the nanoparticle length and based on a study of 14 nanoparticles of widths between 7 and $8 \mathrm{~nm}$ and lengths between 24 and $120 \mathrm{~nm}$, the quasistatic model (which is supposed to work better for low-aspect-ratio NPs) predicts the dipolar plasmonic excitation energies of nanowires with lengths of approximately $25 \pm 5 \mathrm{~nm}<L<45 \pm 5 \mathrm{~nm}$, with a precision of $\Delta E=0.05 \pm 0.05 \mathrm{eV}$, and underestimates (as expected) those of the longer nanowires ( $45 \pm 5 \mathrm{~nm}<L<$ $110 \pm 10 \mathrm{~nm}$ ), with $\Delta E=0.15 \pm 0.05 \mathrm{eV}$, whereas the waveguide model (which is supposed to work better for high-aspect-ratio NPs) allows one to predict the experimental values with a small imprecision over the entire studied nanowire length $L$ range: $\Delta E=-0.05 \pm 0.05 \mathrm{eV}$ for $25 \pm 5 \mathrm{~nm}<L<45 \pm 5 \mathrm{~nm}$, and $\Delta E=0.05 \pm 0.05 \mathrm{eV}$ for $45 \pm 5 \mathrm{~nm}<L<110 \pm 10 \mathrm{~nm}$, as long as retardation effects do not need to be taken into account.

We believe that the experimental verification of the accuracy of numerical and theoretical methods reported here will facilitate the design of alternative plasmonic metamaterials by reducing the need for extensive experimental studies and referring more to numerical simulations, or even to analytical models. Having confirmed that the ion-beam-shaping process produces nanoparticles whose plasmonic properties can be well predicted by theory, and that the experimentally determined plasmonic properties of nanoparticles in a symmetric environment agree well with theory on a nanometric scale, future work is expected to use the ion-beamshaping technique's potential of arranging prolate nanoparticles all aligned in a direction of choice in regular 3D arrays in order to either exploit the plasmonic properties of the isolated nanoparticle on a macroscopic level with widely spaced nanoparticles (with many possible applications in surface-enhanced Raman spectroscopy and second-harmonic generation) or exploit the plasmonic coupling effects between narrowly spaced prolate nanoparticles oriented in a direction of choice, resulting in alternative plasmonic metamaterials (with many possible applications in negative refraction, superimaging, and invisibility cloaking).

\section{ACKNOWLEDGMENTS}

We wish to thank Dr. G. Coddens (LSI, Ecole Polytechnique, CEA) for the many fruitful discussions. Financial support from the French National Research Agency (ANR) for Shaman Project No. ANR-09-BLAN0334, from the METSA network (French Transmission Electron Microscopy and Atom Probe Network), from the French RENATECH network (French national nanofabrication platform), and from Grant No. 274649 from the Academy of Finland is gratefully acknowledged.

[1] S. A. Maier, Plasmonics: Fundamentals and Applications (Springer-Verlag, Berlin, 2007).

[2] L. Novotny and B. Hecht, Principles of Nano-Optics, 2nd ed. (Cambridge University Press, Cambridge, England, 2012).

[3] C. F. Bohren and D. R. Huffman, Absorption and Scattering of Light by Small Particles (John Wiley \& Sons, Hoboken, NJ, 1983).

[4] P. Mühlschlegel, H.-J. Eisler, O. J. F. Martin, B. Hecht, and D. W. Pohl, Resonant optical antennas, Science 308, 1607 (2005).

[5] Kuiru Li, Mark I. Stockman, and David J. Bergman, SelfSimilar Chain of Metal Nanospheres as an Efficient Nanolens, Phys. Rev. Lett. 91, 227402 (2003).

[6] K. L. Kelly, E. Coronado, L. L. Zhao, and G. C. Schatz, The optical properties of metal nanoparticles: The influence of size, shape, and dielectric environment, J. Phys. Chem. B 107, 668 (2003).

[7] G. H. Chan, J. Zhao, E. M. Hicks, G. C. Schatz, and R. P. Van Duyne, Plasmonic properties of copper nanoparticles fabricated by nanosphere lithography, Nano Lett. 7, 1947 (2007).

[8] J. N. Anker, W. P. Hall, O. Lyandres, N. C. Shah, J. Zhao, and R. P. Van Duyne, Biosensing with plasmonic nanosensors, Nat. Mater. 7, 442 (2008).

[9] Amir Weiss and Gilad Haran, Time-dependent singlemolecule Raman scattering as a probe of surface dynamics, J. Phys. Chem. B 105, 12348 (2001).

[10] A. D. McFarland, M. A. Young, J. A. Dieringer, and R. P. Van Duyne, Wavelength-scanned surface-enhanced Raman excitation spectroscopy, J. Phys. Chem. B 109, 11279 (2005)

[11] H. A. Atwater and A. Polman, Plasmonics for improved photovoltaic devices, Nat. Mater. 9, 205 (2010).

[12] S. Kawata, Y. Inouye, and P. Verma, Plasmonics for nearfield nano-imaging and superlensing, Nat. Photonics 3, 388 (2009).

[13] Taiping Zhang, Ségolène Callard, Cécile Jamois, Céline Chevalier, Di Feng, and Ali Belarouci, Plasmonic-photonic crystal coupled nanolaser, Nanotechnology 25, 315201 (2014).

[14] Vladimir M. Shalaev, Wenshan Cai, Uday K. Chettiar, Hsiao-Kuan Yuan, Andrey K. Sarychev, Vladimir P. Drachev, and Alexander V. Kildishev, Negative index of refraction in optical metamaterials, Opt. Lett. 30, 3356 (2005). 
[15] Shuang Zhang, Wenjun Fan, N. C. Panoiu, K. J. Malloy, R. M. Osgood, and S. R. J. Brueck, Experimental Demonstration of Near-Infrared Negative-Index Metamaterials, Phys. Rev. Lett. 95, 137404 (2005).

[16] F. J. Garcia-Vidal, L. Martin-Moreno, and J. B. Pendry, Surfaces with holes in them: New plasmonic metamaterials, J. Opt. A 7, S97 (2005).

[17] Nicholas Fang, Hyesog Lee, Cheng Sun, and Xiang Zhang, Sub-diffraction-limited optical imaging with a silver superlens, Science 308, 534 (2005).

[18] Wenshan Cai, Dentcho A. Genov, and Vladimir M. Shalaev, Superlens based on metal-dielectric composites, Phys. Rev. B 72, 193101 (2005).

[19] Zhaowei Liu, Hyesog Lee, Yi Xiong, Cheng Sun, and Xiang Zhang, Far-field optical hyperlens magnifying subdiffraction-limited objects, Science 315, 1686 (2007).

[20] Zhaowei Liu, Stephane Durant, Hyesog Lee, Yuri Pikus, Nicolas Fang, Yi Xiong, Cheng Sun, and Xiang Zhang, Far-field optical superlens, Nano Lett. 7, 403 (2007).

[21] Wenshan Cai, Uday K. Chettiar, Alexander V. Kildishev, and Vladimir M. Shalaev, Optical cloaking with metamaterials, Nat. Photonics 1, 224 (2007).

[22] Joel Henzie, Min Hyung Lee, and Teri W. Odom, Multiscale patterning of plasmonic metamaterials, Nat. Nanotechnol. 2, 549 (2007).

[23] Juergen Biener, Gregory W. Nyce, Andrea M. Hodge, Monika M. Biener, Alex V. Hamza, and Stefan A. Maier, Nanoporous plasmonic metamaterials, Adv. Mater. 20, 1211 (2008).

[24] Vladimir P. Drachev, Uday K. Chettiar, Alexander V. Kildishev, Hsiao-Kuan Yuan, Wenshan Cai, and Vladimir M. Shalaev, The Ag dielectric function in plasmonic metamaterials, Opt. Express 16, 1186 (2008).

[25] Shuang Zhang, Dentcho A. Genov, Yuan Wang, Ming Liu, and Xiang Zhang, Plasmon-Induced Transparency in Metamaterials, Phys. Rev. Lett. 101, 047401 (2008).

[26] Yoav Avitzour, Yaroslav A. Urzhumov, and Gennady Shvets, Wide-angle infrared absorber based on a negative-index plasmonic metamaterial, Phys. Rev. B 79, 045131 (2009).

[27] K. Tanaka, E. Plum, J. Y. Ou, T. Uchino, and N. I. Zheludev, Multifold Enhancement of Quantum Dot Luminescence in Plasmonic Metamaterials, Phys. Rev. Lett. 105, 227403 (2010).

[28] Alexandra Boltasseva and Harry A. Atwater, Low-loss plasmonic metamaterials, Science 331, 290 (2011).

[29] Jiaming Hao, Lei Zhou, and Min Qiu, Nearly total absorption of light and heat generation by plasmonic metamaterials, Phys. Rev. B 83, 165107 (2011).

[30] Mehdi Keshavarz Hedayati, Mojtaba Javaherirahim, Babak Mozooni, Ramzy Abdelaziz, Ali Tavassolizadeh, Venkata Sai Kiran Chakravadhanula, Vladimir Zaporojtchenko, Thomas Strunkus, Franz Faupel, and Mady Elbahri, Design of a perfect black absorber at visible frequencies using plasmonic metamaterials, Adv. Mater. 23, 5410 (2011).

[31] Gururaj V. Naik and Alexandra Boltasseva, A comparative study of semiconductor-based plasmonic metamaterials, Metamaterials 5, 1 (2011).
[32] Wei Cao, Ranjan Singh, Caihong Zhang, Jiaguang Han, Masayoshi Tonouchi, and Weili Zhang, Plasmon-induced transparency in metamaterials: Active near field coupling between bright superconducting and dark metallic mode resonators, Appl. Phys. Lett. 103, 101106 (2013).

[33] V. G. Kravets, F. Schedin, R. Jalil, L. Britnell, R. V. Gorbachev, D. Ansell, B. Thackray, K. S. Novoselov, A. K. Geim, Andrei V. Kabashin et al., Singular phase nano-optics in plasmonic metamaterials for label-free single-molecule detection, Nat. Mater. 12, 304 (2013).

[34] Wenshan Cai and Vladimir M. Shalaev, Optical Metamaterials (Springer, New York, 2010), Vol. 10.

[35] Hung Ji Huang, Chin Ping Yu, Hung Chun Chang, Kuo Pin Chiu, Hao Ming Chen, Ru Shi Liu, and Din Ping Tsai, Plasmonic optical properties of a single gold nano-rod, Opt. Express 15, 7132 (2007).

[36] Huanjun Chen, Lei Shao, Qian Li, and Jianfang Wang, Gold nanorods and their plasmonic properties, Chem. Soc. Rev. 42, 2679 (2013).

[37] Michael R. Beversluis, Alexandre Bouhelier, and Lukas Novotny, Continuum generation from single gold nanostructures through near-field mediated intraband transitions, Phys. Rev. B 68, 115433 (2003).

[38] Christian Colliex, Mathieu Kociak, and Odile Stéphan, Electron energy loss spectroscopy imaging of surface plasmons at the nanometer scale, Ultramicroscopy $\mathbf{1 6 2}$, A1 (2016).

[39] F. J. García de Abajo and M. Kociak, Probing the Photonic Local Density of States with Electron Energy Loss Spectroscopy, Phys. Rev. Lett. 100, 106804 (2008).

[40] Guillaume Boudarham and Mathieu Kociak, Modal decompositions of the local electromagnetic density of states and spatially resolved electron energy loss probability in terms of geometric modes, Phys. Rev. B 85, 245447 (2012).

[41] J. Nelayah, M. Kociak, O. Stéphan, F. J. G. de Abajo, M. Tencé, L. Henrard, D. Taverna, I. Pastoriza-Santos, L. M. Liz-Marzán, and C. Colliex, Mapping surface plasmons on a single metallic nanoparticle, Nat. Phys. 3, 348 (2007).

[42] S. Mazzucco, O. Stéphan, C. Colliex, I. Pastoriza-Santos, L. M. Liz-Marzan, J. Garcia de Abajo, and M. Kociak, Spatially resolved measurements of plasmonic eigenstates in complex-shaped, asymmetric nanoparticles: Gold nanostars, Eur. Phys. J. Appl. Phys. 54, 33512 (2011).

[43] M. N'Gom, J. Ringnalda, J. F. Mansfield, A. Agarwal, N. Kotov, N. J. Zaluzec, and T. B. Norris, Single particle plasmon spectroscopy of silver nanowires and gold nanorods, Nano Lett. 8, 3200 (2008).

[44] M. Bosman, V. J. Keast, M. Watanabe, A. I. Maaroof, and M. B. Cortie, Mapping surface plasmons at the nanometre scale with an electron beam, Nanotechnology 18, 165505 (2007).

[45] S. Mazzucco, N. Geuquet, J. Ye, O. Stéphan, W. Van Roy, P. Van Dorpe, L. Henrard, and M. Kociak, Ultra local modification of surface plasmons properties in silver nanocubes, Nano Lett. 12, 1288 (2012).

[46] D. Rossouw, M. Couillard, J. Vickery, E. Kumacheva, and G. A. Botton, Multipolar plasmonic resonances in silver 
nanowire antennas imaged with a subnanometer electron probe, Nano Lett. 11, 1499 (2011).

[47] B. S. Guiton, V. Iberi, S. Li, D. N. Leonard, C. M. Parish, P. G. Kotula, M. Varela, G. C. Schatz, S. J. Pennycook, and J.P. Camden, Correlated optical measurements and plasmon mapping of silver nanorods, Nano Lett. 11, 3482 (2011).

[48] I. Alber, W. Sigle, S. Müller, R. Neumann, O. Picht, M. Rauber, P. A. van Aken, and M. E. Toimil-Molares, Visualization of multipolar longitudinal and transversal surface plasmon modes in nanowire dimers, ACS Nano 5, 9845 (2011).

[49] Michel Bosman, Lei Zhang, Huigao Duan, Shu Fen Tan, Christian A. Nijhuis, Cheng-Wei Qiu, and Joel K. W. Yang, Encapsulated annealing: Enhancing the plasmon quality factor in lithographically-defined nanostructures, Sci. Rep. 4, 5537 (2014).

[50] Sujit Kumar Ghosh, Sudip Nath, Subrata Kundu, Kunio Esumi, and Tarasankar Pal, Solvent and ligand effects on the localized surface plasmon resonance (LSPR) of gold colloids, J. Phys. Chem. B 108, 13963 (2004).

[51] Mark W. Knight, Yanpeng Wu, J. Britt Lassiter, Peter Nordlander, and Naomi J. Halas, Substrates matter: Influence of an adjacent dielectric on an individual plasmonic nanoparticle, Nano Lett. 9, 2188 (2009).

[52] Shunping Zhang, Kui Bao, Naomi J. Halas, Hongxing Xu, and Peter Nordlander, Substrate-induced Fano resonances of a plasmonic nanocube: A route to increased-sensitivity localized surface plasmon resonance sensors revealed, Nano Lett. 11, 1657 (2011).

[53] C. D’Orleans, J. P. Stoquert, C. Estournes, C. Cerruti, J. J. Grob, J. L. Guille, F. Haas, D. Muller, and M. RichardPlouet, Anisotropy of Co nanoparticles induced by swift heavy ions, Phys. Rev. B 67, 220101 (2003).

[54] Sjoerd Roorda, Teun van Dillen, Albert Polman, Christina Graf, Alphons van Blaaderen, and Bart J. Kooi, Aligned gold nanorods in silica made by ion irradiation o f core-shell colloidal particles, Adv. Mater. 16, 235 (2004).

[55] Joan J. Penninkhof, Christina Graf, Teun van Dillen, Arjen M. Vredenberg, Alfons van Blaaderen, and Albert Polman, Angle-dependent extinction of anisotropic silica/Au core/ shell colloids made via ion irradiation, Adv. Mater. 17, 1484 (2005).

[56] J. J. Penninkhof, T. Van Dillen, S. Roorda, C. Graf, A. Van Blaaderen, A. M. Vredenberg, and A. Polman, Anisotropic deformation of metallo-dielectric core-shell colloids under $\mathrm{MeV}$ ion irradiation, Nucl. Instrum. Methods Phys. Res., Sect. B 242, 523 (2006).

[57] A. Oliver, J. A. Reyes-Esqueda, J. C. Cheang-Wong, C. E. Román-Velázquez, A. Crespo-Sosa, L. Rodriguez-Fernandez, J. A. Seman, and Cecilia Noguez, Controlled anisotropic deformation of $\mathrm{Ag}$ nanoparticles by $\mathrm{Si}$ ion irradiation, Phys. Rev. B 74, 245425 (2006).

[58] Y. K. Mishra, F. Singh, D. K. Avasthi, J. C. Pivin, D. Malinovska, and E. Pippel, Synthesis of elongated Au nanoparticles in silica matrix by ion irradiation, Appl. Phys. Lett. 91, 063103 (2007).

[59] Koichi Awazu, Xiaomin Wang, Makoto Fujimaki, Junji Tominaga, Hirohiko Aiba, Yoshimichi Ohki, and Tetsuro
Komatsubara, Elongation of gold nanoparticles in silica glass by irradiation with swift heavy ions, Phys. Rev. B 78, 054102 (2008).

[60] R. Giulian, P. Kluth, L. L. Araujo, D. J. Sprouster, A. P. Byrne, D. J. Cookson, and M. C. Ridgway, Shape transformation of Pt nanoparticles induced by swift heavy-ion irradiation, Phys. Rev. B 78, 125413 (2008).

[61] E. A. Dawi, G. Rizza, M. P. Mink, A. M. Vredenberg, and F. Habraken, Ion beam shaping of $\mathrm{Au}$ nanoparticles in silica: Particle size and concentration dependence, J. Appl. Phys. 105, 074305 (2009).

[62] Jorge Alejandro Reyes-Esqueda, Vladimir RodríguezIglesias, Héctor-Gabriel Silva-Pereyra, Carlos TorresTorres, Ana-Laura Santiago-Ramírez, Juan Carlos Cheang-Wong, Alejandro Crespo-Sosa, Luis RodríguezFernández, Alejandra López-Suárez, and Alicia Oliver, Anisotropic linear and nonlinear optical properties from anisotropy-controlled metallic nanocomposites, Opt. Express 17, 12849 (2009).

[63] Vladimir Rodriguez-Iglesias, O. Pena-Rodriguez, Hector Gabriel Silva-Pereyra, Luis Rodriguez-Fernandez, Guinther Kellermann, Juan Carlos Cheang-Wong, Alejandro Crespo-Sosa, and Alicia Oliver, Elongated gold nanoparticles obtained by ion implantation in silica: Characterization and $T$-matrix simulations, J. Phys. Chem. C 114, 746 (2010).

[64] Mark C. Ridgway, Patrick Kluth, Raquel Giulian, D. J. Sprouster, L. L. Araujo, C. S. Schnohr, D. J. Llewellyn, A. P. Byrne, Garry J. Foran, and D. J. Cookson, Changes in metal nanoparticle shape and size induced by swift heavyion irradiation, Nucl. Instrum. Methods Phys. Res., Sect. B 267, 931 (2009).

[65] G. Rizza, E. A. Dawi, A. M. Vredenberg, and Isabelle Monnet, Ion engineering of embedded nanostructures: From spherical to facetted nanoparticles, Appl. Phys. Lett. 95, 043105 (2009).

[66] D. K. Avasthi, Y. K. Mishra, F. Singh, and J. P. Stoquert, Ion tracks in silica for engineering the embedded nanoparticles, Nucl. Instrum. Methods Phys. Res., Sect. B 268, 3027 (2010).

[67] Giancarlo Rizza, Farah Attouchi, Pierre-Eugene Coulon, Sandrine Perruchas, Thierry Gacoin, Isabelle Monnet, and Ludovic Largeau, Rayleigh-like instability in the ion-shaping of Au-Ag alloy nanoparticles embedded within a silica matrix, Nanotechnology 22, 175305 (2011).

[68] D. J. Sprouster, R. Giulian, L. L. Araujo, P. Kluth, B. Johannessen, D. J. Cookson, and M. C. Ridgway, Swift heavy-ion irradiation-induced shape and structural transformation in cobalt nanoparticles, J. Appl. Phys. 109, 113504 (2011).

[69] E. A. Dawi, A. M. Vredenberg, G. Rizza, and Marcel Toulemonde, Ion-induced elongation of gold nanoparticles in silica by irradiation with $\mathrm{Ag}$ and $\mathrm{Cu}$ swift heavy ions: Track radius and energy loss threshold, Nanotechnology 22, 215607 (2011).

[70] Mark C. Ridgway, Raquel Giulian, David J. Sprouster, Patrick Kluth, Leandro Langie Araújo, D. J. Llewellyn, A. P. Byrne, Felipe Kremer, Paulo Fernando Papaleo Fichtner, G. Rizza et al., Role of Thermodynamics in the Shape 
Transformation of Embedded Metal Nanoparticles Induced by Swift Heavy-Ion Irradiation, Phys. Rev. Lett. 106, 095505 (2011).

[71] G. Rizza, P. E. Coulon, V. Khomenkov, C. Dufour, I. Monnet, M. Toulemonde, S. Perruchas, T. Gacoin, D. Mailly, X. Lafosse et al., Rational description of the ionbeam shaping mechanism, Phys. Rev. B 86, 035450 (2012).

[72] Ch. Dufour, V. Khomenkov, G. Rizza, and M. Toulemonde, Ion-matter interaction: The three-dimensional version of the thermal spike model. Application to nanoparticle irradiation with swift heavy ions, J. Phys. D 45, 065302 (2012).

[73] H. Amekura, S. Mohapatra, U. B. Singh, S. A. Khan, P. K. Kulriya, N. Ishikawa, N. Okubo, and D. K. Avasthi, Shape elongation of $\mathrm{Zn}$ nanoparticles in silica irradiated with swift heavy ions of different species and energies: Scaling law and some insights on the elongation mechanism, Nanotechnology 25, 435301 (2014).

[74] Aleksi A. Leino, Flyura Djurabekova, and Kai Nordlund, Radiation effects in nanoclusters embedded in solids, Eur. Phys. J. B 87, 242 (2014).

[75] Mark C. Ridgway, Flyura Djurabekova, and Kai Nordlund, Ion-solid interactions at the extremes of electronic energy loss: Examples for amorphous semiconductors and embedded nanostructures, Curr. Opin. Solid State Mater. Sci. 19, 29 (2015).

[76] Giancarlo Rizza, From ion-hammering to ion-shaping: An historical overview, J. Phys. Conf. Ser. 629, 012005 (2015).

[77] Pierre-Eugene Coulon, Julia Amici, Marie-Claude Clochard, Vladimir Khomenkov, Christian Dufour, Isabelle Monnet, Clara Grygiel, Sandrine Perruchas, Christian Ulysse, Ludovic Largeau et al., Ion-shaping of embedded gold hollow nanoshells into vertically aligned prolate morphologies, Sci. Rep. 6, 21116 (2016).

[78] See Supplemental Material at http://link.aps.org/ supplemental/10.1103/PhysRevApplied.9.064038 for further details on the experimental and numerical methods, the wave-vector $\beta$ determination, and the analytical models.

[79] Enric Garcia-Caurel, Antonello De Martino, Jean-Paul Gaston, and Li Yan, Application of spectroscopic ellipsometry and Mueller ellipsometry to optical characterization, Appl. Spectrosc. 67, 1 (2013).

[80] J. F. Ziegler, J. P. Biersack, and U. Littmark, The Stopping Ranges and Ranges of Ions in Solids (Pergamon Press, New York, 1985). The simulation code is available at http://www.srim.org.

[81] C. Jeanguillaume and C. Colliex, Spectrum-image: The next step in EELS digital acquisition and processing, Ultramicroscopy 28, 252 (1989).

[82] F. Hao, C. L. Nehl, J. H. Hafner, and P. Nordlander, Plasmon resonances of a gold nanostar, Nano Lett. 7, 729 (2007).

[83] V. Myroshnychenko, J. Rodriguez-Fernandez, I. PastorizaSantos, A. M. Funston, C. Novo, P. Mulvaney, L. M. LizMarzan, and F. J. Garcia de Abajo, Modelling the optical response of gold nanoparticles, Chem. Soc. Rev. 37, 1792 (2008).

[84] J. Dorfmuller, R. Vogelgesang, W. Khunsin, C. Rockstuhl, C. Etrich, and K. Kern, Plasmonic nanowire antennas:
Experiment, simulation, and theory, Nano Lett. 10, 3596 (2010).

[85] A. Taflove and S. C. Hagness, Computational Electrodynamics: The Finite-Difference Time-Domain Method (Artech House, Norwood, MA, 2000).

[86] W. H. P. Pernice, F. P. Payne, and D. F. G. Gallagher, An FDTD method for the simulation of dispersive metallic structures, Opt. Quantum Electron. 38, 843 (2007).

[87] A. Vial, A.-S. Grimault, D. Macías, D. Barchiesi, and M. Lamy de La Chapelle, Improved analytical fit of gold dispersion: Application to the modeling of extinction spectra with a finite-difference time-domain method, Phys. Rev. B 71, 085416 (2005).

[88] Jean-Pierre Berenger, A perfectly matched layer for the absorption of electromagnetic waves, J. Comput. Phys. 114, 185 (1994).

[89] Ulrich Hohenester, Simulating electron energy loss spectroscopy with the MNPBEM toolbox, Comput. Phys. Commun. 185, 1177 (2014).

[90] Ulrich Hohenester and Andreas Truegler, MNPBEM-A MATLAB toolbox for the simulation of plasmonic nanoparticles, Comput. Phys. Commun. 183, 370 (2012).

[91] Jurgen Waxenegger, Andreas Trugler, and Ulrich Hohenester, Plasmonics simulations with the MNPBEM toolbox: Consideration of substrates and layer structures, Comput. Phys. Commun. 193, 138 (2015).

[92] Irene Ament, Janak Prasad, Andreas Henkel, Sebastian Schmachtel, and Carsten Sonnichsen, Single unlabeled protein detection on individual plasmonic nanoparticles, Nano Lett. 12, 1092 (2012).

[93] Tobias Hanke, Julijan Cesar, Vanessa Knittel, Andreas Trugler, Ulrich Hohenester, Alfred Leitenstorfer, and Rudolf Bratschitsch, Tailoring spatiotemporal light confinement in single plasmonic nanoantennas, Nano Lett. 12, 992 (2012).

[94] Franz-Philipp Schmidt, Harald Ditlbacher, Ulrich Hohenester, Andreas Hohenau, Ferdinand Hofer, and Joachim R. Krenn, Dark plasmonic breathing modes in silver nanodisks, Nano Lett. 12, 5780 (2012).

[95] Peter Dombi, Anton Horl, Peter Racz, Istvan Marton, Andreas Trugler, Joachim R. Krenn, and Ulrich Hohenester, Ultrafast strong-field photoemission from plasmonic nanoparticles, Nano Lett. 13, 674 (2013).

[96] Christian Gruber, Andreas Trugler, Andreas Hohenau, Ulrich Hohenester, and Joachim R. Krenn, Spectral modifications and polarization dependent coupling in tailored assemblies of quantum dots and plasmonic nanowires, Nano Lett. 13, 4257 (2013).

[97] Anton Horl, Andreas Trugler, and Ulrich Hohenester, Tomography of Particle Plasmon Fields from Electron Energy Loss Spectroscopy, Phys. Rev. Lett. 111, 076801 (2013).

[98] Yunuen Montelongo, Jaime O. Tenorio-Pearl, William I. Milne, and Timothy D. Wilkinson, Polarization switchable diffraction based on subwavelength plasmonic nanoantennas, Nano Lett. 14, 294 (2014).

[99] David Rossouw and Gianluigi A. Botton, Plasmonic Response of Bent Silver Nanowires for Nanophotonic Sub- 
wavelength Waveguiding, Phys. Rev. Lett. 110, 066801 (2013).

[100] Bart Goris, Giulio Guzzinati, Cristina FernandezLopez, Jorge Perez-Juste, Luis M. Liz-Marzan, Andreas Trugler, Ulrich Hohenester, Jo Verbeeck, Sara Bals, and Gustaaf Van Tendeloo, Plasmon mapping in Au@Ag nanocube assemblies, J. Phys. Chem. C 118, 15356 (2014).

[101] Christoph Huber, Andreas Trügler, Ulrich Hohenester, Yehiam Prior, and Wolfgang Kautek, Optical near-field excitation at commercial scanning probe microscopy tips: A theoretical and experimental investigation, Phys. Chem. Chem. Phys. 16, 2289 (2014).

[102] Jérôme Martin, Mathieu Kociak, Zackaria Mahfoud, Julien Proust, Davy Gérard, and Jérôme Plain, High-resolution imaging and spectroscopy of multipolar plasmonic resonances in aluminum nanoantennas, Nano Lett. 14, 5517 (2014).

[103] Owen D. Miller, Chia W. Hsu, M. T. Homer Reid, Wenjun Qiu, Brendan G. DeLacy, John D. Joannopoulos, Marin Soljačić, and Steven G. Johnson, Fundamental Limits to Extinction by Metallic Nanoparticles, Phys. Rev. Lett. 112, 123903 (2014).

[104] Franz P. Schmidt, Harald Ditlbacher, Ferdinand Hofer, Joachim R. Krenn, and Ulrich Hohenester, Morphing a plasmonic nanodisk into a nanotriangle, Nano Lett. 14, 4810 (2014).

[105] Franz-Philipp Schmidt, Harald Ditlbacher, Ulrich Hohenester, Andreas Hohenau, Ferdinand Hofer, and Joachim R. Krenn, Universal dispersion of surface plasmons in flat nanostructures, Nat. Commun. 5, 3604 (2014).

[106] Wei Sun, Etienne Boulais, Yera Hakobyan, Wei Li Wang, Amy Guan, Mark Bathe, and Peng Yin, Casting inorganic structures with DNA molds, Science 346, 1258361 (2014).

[107] Jakob Ebner, Andreas Trugler, and Ulrich Hohenester, Optical excitations of hybrid metal-semiconductor nanoparticles, Eur. Phys. J. B 88, 11 (2015).

[108] Dario Knebl, Anton Horl, Andreas Trugler, Johannes Kern, Joachim R. Krenn, Peter Puschnig, and Ulrich Hohenester, Gap plasmonics of silver nanocube dimers, Phys. Rev. B 93, 081405 (2016).

[109] F. J. Garcia de Abajo and A. Howie, Retarded field calculation of electron energy loss in inhomogeneous dielectrics, Phys. Rev. B 65, 115418 (2002).

[110] M. Pelton and G. W. Bryant, Introduction to Metal-Nanoparticle Plasmonics (John Wiley \& Sons, Hoboken, NJ, 2013).

[111] Edward D. Palik, Handbook of Optical Constants of Solids (Academic Press, New York, 1998), Vol. 3.

[112] Julien Cardin and Dominique Leduc, Determination of refractive index, thickness, and the optical losses of thin films from prism-film coupling measurements, Appl. Opt. 47, 894 (2008).

[113] F. J. Garcia de Abajo, Optical excitations in electron microscopy, Rev. Mod. Phys. 82, 209 (2010).

[114] Per-Olof Persson and Gilbert Strang, A simple mesh generator in MATLAB, SIAM Rev. 46, 329 (2004).

[115] Jeffrey M. McMahon, Stephen K. Gray, and George C. Schatz, Nonlocal Optical Response of Metal
Nanostructures with Arbitrary Shape, Phys. Rev. Lett. 103, 097403 (2009).

[116] C. Sönnichsen, T. Franzl, T. Wilk, G. von Plessen, J. Feldmann, O. Wilson, and P. Mulvaney, Drastic Reduction of Plasmon Damping in Gold Nanorods, Phys. Rev. Lett. 88, 077402 (2002).

[117] U. Kreibig and M. Vollmer, Optical Properties of Metal Clusters (Springer, Berlin, 1995).

[118] P. B. Johnson and R. W. Christy, Optical constants of the noble metals, Phys. Rev. B 6, 4370 (1972).

[119] Ernst Jan R. Vesseur, René de Waele, Martin Kuttge, and Albert Polman, Direct observation of plasmonic modes in $\mathrm{Au}$ nanowires using high-resolution cathodoluminescence spectroscopy, Nano Lett. 7, 2843 (2007).

[120] Mathieu Kociak and Odile Stéphan, Mapping plasmons at the nanometer scale in an electron microscope, Chem. Soc. Rev. 43, 3865 (2014).

[121] S. Eustis and M. A. El-Sayed, Determination of the aspect ratio statistical distribution of gold nanorods in solution from a theoretical fit of the observed inhomogeneously broadened longitudinal plasmon resonance absorption spectrum, J. Appl. Phys. 100, 044324 (2006).

[122] S. Link, M. B. Mohamed, and M. A. El-Sayed, Simulation of the optical absorption spectra of gold nanorods as a function of their aspect ratio and the effect of the medium dielectric constant, J. Phys. Chem. B 103, 3073 (1999).

[123] S. Link and M. A. El-Sayed, Simulation of the optical absorption spectra of gold nanorods as a function of their aspect ratio and the effect of the medium dielectric constant, J. Phys. Chem. B 109, 10531 (2005).

[124] Shakeeb Bin Hasan, Robert Filter, Aftab Ahmed, Ralf Vogelgesang, Reuven Gordon, Carsten Rockstuhl, and Falk Lederer, Relating localized nanoparticle resonances to an associated antenna problem, Phys. Rev. B 84, 195405 (2011).

[125] David T. Schoen, Ashwin C. Atre, Aitzol García-Etxarri, Jennifer A. Dionne, and Mark L. Brongersma, Probing complex reflection coefficients in one-dimensional surface plasmon polariton waveguides and cavities using stem EELS, Nano Lett. 15, 120 (2015).

[126] A. L. Gonzalez, J. A. Reyes-Esqueda, and Cecilia Noguez, Optical properties of elongated noble metal nanoparticles, J. Phys. Chem. C 112, 7356 (2008).

[127] I. N. Bronstein, K. A. Semendjajew, G. Musiol, and H. Mühlig, Taschenbuch der Mathematik (Verlag Harri Deutsch, Frankfurt, 1999).

[128] J. A. Stratton, Electromagnetic Theory (McGraw-Hill, New York, 1941).

[129] B. E. Sernelius, Surface Modes in Physics (Wiley-VCH, Weinheim, 2011).

[130] C. A. Pfeiffer, E. N. Economou, and K. L. Ngai, Surface polaritons in a circularly cylindrical interface: Surface plasmons, Phys. Rev. B 10, 3038 (1974).

[131] Jukka Venermo and Ari Sihvola, Dielectric polarizability of circular cylinder, J. Electrost. 63, 101 (2005).

[132] Nanfang Yu, Patrice Genevet, Mikhail A. Kats, Francesco Aieta, Jean-Philippe Tetienne, Federico Capasso, and Zeno Gaburro, Light propagation with phase discontinuities: 
Generalized laws of reflection and refraction, Science 334, 333 (2011).

[133] Sebastian Schlücker, Surface-enhanced raman spectroscopy: Concepts and chemical applications, Angew. Chem., Int. Ed. Engl. 53, 4756 (2014).

[134] G. Vecchi, V. Giannini, and J. Gómez Rivas, Surface modes in plasmonic crystals induced by diffractive coupling of nanoantennas, Phys. Rev. B 80, 201401 (2009).

[135] Ugo Fano, Effects of configuration interaction on intensities and phase shifts, Phys. Rev. 124, 1866 (1961).

[136] K. T. Carron, W. Fluhr, M. Meier, A. Wokaun, and H. W. Lehmann, Resonances of two-dimensional particle gratings in surface-enhanced Raman scattering, J. Opt. Soc. Am. B 3, 430 (1986).

[137] A. Christ, Y. Ekinci, H. H. Solak, N. A. Gippius, S. G. Tikhodeev, and O.J.F. Martin, Controlling the Fano interference in a plasmonic lattice, Phys. Rev. B 76, 201405 (2007).

[138] Said Rahimzadeh Kalaleh Rodriguez, Aimi Abass, Björn Maes, Olaf T. A. Janssen, Gabriele Vecchi, and J. Gómez Rivas, Coupling Bright and Dark Plasmonic Lattice Resonances, Phys. Rev. X 1, 021019 (2011).

[139] Dmitry Khlopin, Frédéric Laux, William P. Wardley, Jérôme Martin, Gregory A. Wurtz, Jérôme Plain, Nicolas Bonod, Anatoly V. Zayats, Wayne Dickson, and Davy Gérard, Lattice modes and plasmonic linewidth engineering in gold and aluminum nanoparticle arrays, J. Opt. Soc. Am. B 34, 691 (2017).
[140] Abdallah Slablab, Tero J. Isotalo, Jouni Mäkitalo, Léo Turquet, Pierre-Eugène Coulon, Tapio Niemi, Christian Ulysse, Mathieu Kociak, Dominique Mailly, Giancarlo Rizza, and Martti Kauranen, Fabrication of ion-shaped anisotropic nanoparticles and their orientational imaging by second-harmonic generation microscopy, Sci. Rep. 6, 37469 (2016).

[141] Creative Commons CC BY license, https:// creativecommons.org/licenses/by/4.0/.

[142] R. W. Boyd, Nonlinear Optics, 3rd ed. (Academic Press, Burlington, MA, 2008).

[143] Alexander Poddubny, Ivan Iorsh, Pavel Belov, and Yuri Kivshar, Hyperbolic metamaterials, Nat. Photonics 7, 948 (2013).

[144] Lorenzo Ferrari, Chihhui Wu, Dominic Lepage, Xiang Zhang, and Zhaowei Liu, Hyperbolic metamaterials and their applications, Prog. Quantum Electron. 40, 1 (2015).

[145] Jason Valentine, Shuang Zhang, Thomas Zentgraf, Erick Ulin-Avila, Dentcho A. Genov, Guy Bartal, and Xiang Zhang, Three-dimensional optical metamaterial with a negative refractive index, Nature (London) 455, 376 (2008).

[146] Tolga Ergin, Nicolas Stenger, Patrice Brenner, John B. Pendry, and Martin Wegener, Three-dimensional invisibility cloak at optical wavelengths, Science 328, 337 (2010). 